NISTIR 7238

\title{
Investigation of the Impact of Commercial Building Envelope Airtightness on HVAC Energy Use
}

Steven J. Emmerich

Tim McDowell

Wagdy Anis

National Institute of Standards and Technology

Technology Administration, U.S. Department of Commerce 


\section{Investigation of the Impact of Commercial Building Envelope Airtightness on HVAC Energy Use}

Steven J. Emmerich

Building and Fire Research Laboratory

Timothy P. McDowell TESS, Inc.

Wagdy Anis Shepley Bulfinch Richardson and Abbott

Prepared for:

U.S. Department of Energy Office of Building Technologies

June 2005

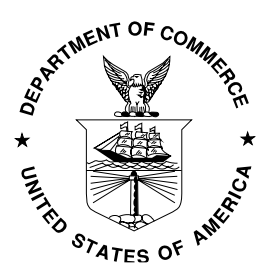

U.S. Department of Commerce Carlos M. Gutierrez, Secretary

Technology Administration

Phillip J. Bond, Under Secretary of Commerce for Technology National Institute of Standards and Technology 


\begin{abstract}
This report presents a simulation study of the energy impact of improving envelope airtightness in U.S. commercial buildings. Despite common assumptions, measurements have shown that typical U.S. commercial buildings are not particularly airtight. Past simulation studies have shown that commercial building envelope leakage can result in significant heating and cooling loads. To evaluate the potential energy savings of an effective air barrier requirement, annual energy simulations were prepared for three nonresidential buildings (a two-story office building, a one-story retail building, and a four-story apartment building) in 5 U.S. cities. A coupled multizone airflow and building energy simulation tool was used to predict the energy use for the buildings at a target tightness level relative to a baseline level based on measurements in existing buildings. Based on assumed blended national average heating and cooling energy prices, predicted potential annual heating and cooling energy cost savings ranged from $3 \%$ to $36 \%$ with the smallest savings occurring in the cooling-dominated climates of Phoenix and Miami. In order to put these estimated energy savings in context, a cost effectiveness calculation was performed using the scalar ratio methodology employed by ASHRAE SSPC 90.1.
\end{abstract}

Keywords: energy efficiency, infiltration, office buildings, ventilation 


\section{TABLE OF CONTENTS}

ABSTRACT__ iii

INTRODUCTION__ 1

ANALYSIS METHOD___ 2

BUILDING DESCRIPTIONS _ _ 3

AIRFLOW MODELS_ 16

SYSTEM MODELS___ 18

SCALAR CALCULATION__ 19

RESULTS _ 21

DISCUSSION__ 34

ACKNOWLEDGEMENTS _ 35

REFERENCES _ 36

Appendix A: Commercial Building Airtightness Data___ 39 


\section{Introduction}

The objective of this study is to investigate the impact of envelope airtightness on the energy consumption of typical commercial buildings in the U.S. Despite common assumptions that envelope air leakage is not significant in office and other commercial buildings, measurements have shown that these buildings are subject to larger infiltration rates than commonly believed (Persily 1998, Proskiw and Phillips 2001). Infiltration in commercial buildings can have many negative consequences, including reduced thermal comfort, interference with the proper operation of mechanical ventilation systems, degraded indoor air quality, moisture damage of building envelope components, and increased energy consumption. For these reasons, attention has been given to methods of improving airtightness both in existing buildings and new constructions (Persily 1993). Since 1997, the Building Environment and Thermal Envelope Council of the National Institute of Building Sciences has sponsored several symposia in the U.S. on the topic of air barriers for buildings in North American climates. Canada Mortgage and Housing Corporation has sponsored similar conferences in Canada. Others have also published articles on the importance of air leakage in commercial buildings (Anis 2001, Ask 2003). However, the focus of these conferences and publications has largely been air barrier technology and the non-energy impacts of air leakage in buildings. In order to evaluate the cost effectiveness of such measures to tighten buildings, estimates of the impact of air leakage on energy use are needed.

An earlier study estimated the national impact of infiltration in office buildings based on a simplified method for calculating both the infiltration flows and the building energy use (Emmerich et al. 1995). The loads were calculated for a set of 25 buildings, each representing a certain percentage of the total office building stock of the United States. Twenty of these buildings represent the existing office building stock as of 1979 (Briggs, Crawley, and Schliesing 1992) and five represent construction between 1980 and 1995 (Crawley and Schliesing 1992). Further work improved on this initial method by using airflows from multizone airflow simulations (Emmerich and Persily 1998) combined with a simple load calculation. More recently, a more detailed analysis method to determine the impact of infiltration and ventilation rates on building energy usage was developed (McDowell et al. 2003). This approach included the coupling of a detailed multi-zone airflow model based on the CONTAMW model (Dols and Walton 2002) and the detailed multi-zone building energy modeling program TRNSYS (Klein 2000). This project demonstrated the ability of the coupled programs to study the annual heating and cooling energy use in the US office building stock as a function of infiltration and ventilation rates.

The American Society of Heating, Refrigerating, and Air-Conditioning Engineers (ASHRAE) Standard 90.1 Envelope Subcommittee has formed a task group to consider updating the building air leakage requirements in the standard to require a continuous air barrier system. An air barrier system is the combination of interconnected materials, flexible joint systems, and components of the building envelope that provide the air-tightness of the building. Included in the current standard are detailed quantitative limits for air leakage through fenestration and doors but only general qualitative guidance for the opaque portion of the building envelope (ASHRAE 2001b). For example, the Standard requires sealing, caulking, gasketing, or weather-stripping such locations as joints around fenestration and doors, junctions between floors, walls, and roofs, etc. However, there is no quantitative air leakage limit specified for either the wall and other envelope components or the building as a whole. This is analogous to requiring that care be taken when installing insulation without requiring any minimum R-value. 


\section{Analysis Method}

To provide input to the ASHRAE 90.1 Envelope Subcommittee in its consideration of the potential energy savings and cost effectiveness of an effective air barrier requirement, annual energy simulations and cost estimates were prepared for three common, modern nonresidential buildings - a two-story office building, a one-story retail building, and a four-story apartment building. The apartment building is included because the scope of Standard 90.1 includes multi-family structures of more than three stories above grade. The new combined airflow-building energy modeling tool (described by McDowell et al. 2003) was used to estimate the energy impact of envelope airtightness in multiple U.S. climate types. HVAC systems representative of the types used in these buildings were included in the building models. Other building model parameters were chosen such that the buildings would be considered typical new construction and meet current ASHRAE Standard 90.1 requirements.

Energy simulations were performed using TRNSYS (Klein 2000) - a transient system simulation program with a modular structure that was designed to solve complex energy system problems by dividing the problem into a series of smaller components. Each of these components can then be solved independently and coupled with other components to simulate and solve the larger system problem. Components (or Types as they are called) in TRNSYS may be as simple as a pump or pipe, or as complicated as a multizone building model. The entire program is then a collection of energy system component models grouped around a simulation engine (solver). The modular nature of the program makes it easier to add content to the program by introducing new component models to the standard package. The simulation engine provides the capability of interconnecting system components in any desired manner, solving differential equations, and facilitating inputs and outputs. The TRNSYS multi-zone building model (called Type 56) includes heat transfer by conduction, convection and radiation, heat gains due to the presence of occupants and equipment, and the storage of heat in the room air and building mass.

The infiltration in the buildings was modeled using a TRNSYS type based on an updated version of the AIRNET model (Walton 1989), which is included in the multizone airflow and contaminant dispersal program CONTAMW (Dols and Walton 2002). CONTAMW combines the best available algorithms for modeling airflow and contaminant transport in multizone buildings with a graphic interface for data input and display of results. The multizone approach is implemented by constructing a network of elements describing the flow paths (HVAC ducts, doors, windows, cracks, etc.) between the zones of a building. The network nodes represent the zones, each of which are modeled at a uniform temperature and pollutant concentration. The pressures vary hydrostatically, so the zone pressure values are a function of the elevation within the zone. The network of equations is then solved at each time step of the simulation. McDowell et al. (2003) described the coupling of the TRNSYS and CONTAM models.

Simulations of annual energy use were run using TMY2 files (Marion and Urban 1995) for five different cities representing different climate zones of the US (Miami, Phoenix, St. Louis, Bismarck, and Minneapolis) and at three levels of airtightness representing different construction practices. The levels of airtightness were selected to represent 1) no air barrier, 2) target air barrier, and 3) best achievable levels through a review of measured commercial building airtightness data (Persily 1998), ASHRAE Handbook data (ASHRAE 2001c) and other sources. Each building was modeled once with frame construction and then masonry construction. Thus, the matrix of simulations is 3 building types $\mathrm{X} 2$ envelope construction types X 3 airtightness levels X 5 climates, for a total of 90 simulation cases. 


\section{Building Descriptions}

This section describes the three buildings modeled in the study.

\section{Thermal Properties, Setpoints, and Schedules}

The building models were developed so that the wall constructions and windows would satisfy the requirements of ASHRAE Standard 90.1 (ASHRAE 90.1-2001b) for the different locations as described below.

\section{Office Building}

The building modeled is a two story office building with a total floor area of $2250 \mathrm{~m} 2(24,200 \mathrm{ft} 2)$ and a floorplan as shown in Figure 1. The building has a window-to-wall ratio of 0.2 with a floor-to-floor height

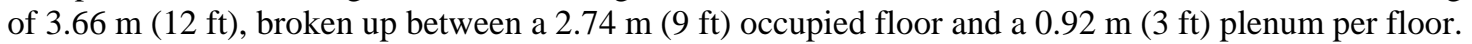
The building also includes a single elevator shaft.
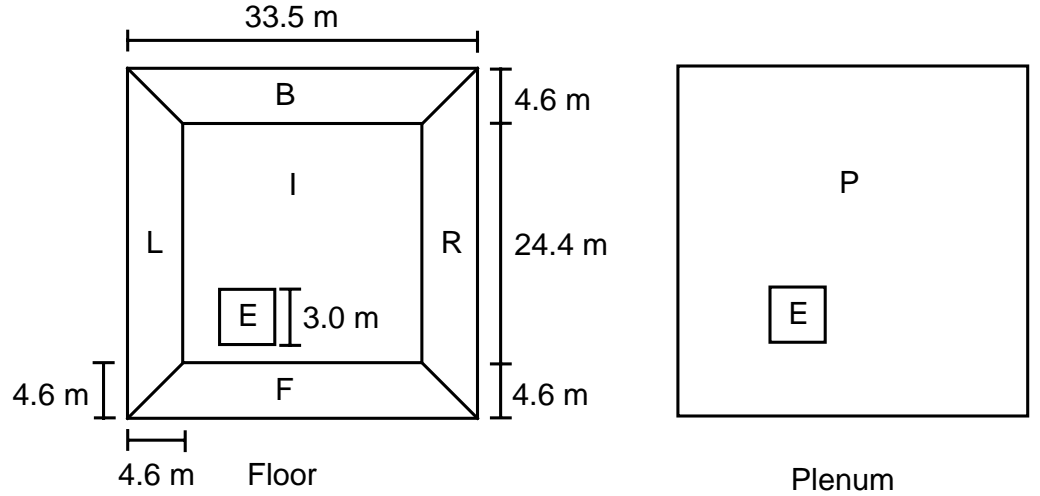

\begin{tabular}{|c|c}
\hline Plenum & $0.9 \mathrm{~m}$ \\
\cline { 1 - 1 } Floor 2 & $2.7 \mathrm{~m}$ \\
\cline { 1 - 1 } Plenum & $0.9 \mathrm{~m}$ \\
\cline { 1 - 1 } Floor 1 & $2.7 \mathrm{~m}$
\end{tabular}

$F, R, B, L$ are the front, right, back, and left perimeter zones.

$I$ is the core zone. $E$ is the elevator zone.

\section{Figure 1 Floorplan of two-story office building}

The wall, roof, slab and window thermal properties used for modeling the office building are summarized in Table 1.

Table 1 Wall, roof, slab and window thermal properties for office building

Frame Wall Construction:

\begin{tabular}{|c|c|c|c|c|c|}
\hline St. Louis, Miami and Phoenix & Thickness & Conductivity & Density & Specific Heat & Resistance \\
\hline Description & $\begin{array}{c}\mathrm{m} \\
(\mathrm{ft})\end{array}$ & $\begin{array}{c}\mathrm{W} / \mathrm{m}-\mathrm{K} \\
(\mathrm{Btu} / \mathrm{hr}-\mathrm{ft}-\mathrm{F})\end{array}$ & $\begin{array}{l}\mathrm{kg} / \mathrm{m}^{3} \\
\left(\mathrm{lb} / \mathrm{ft}^{3}\right)\end{array}$ & $\begin{array}{c}\mathrm{kJ} / \mathrm{kg}-\mathrm{K} \\
(\mathrm{Btu} / \mathrm{lb}-\mathrm{F})\end{array}$ & $\begin{array}{c}\mathrm{m}^{2}-\mathrm{K} / \mathrm{W} \\
\left(\mathrm{hr}-\mathrm{ft}^{2}-\mathrm{F} / \mathrm{Btu}\right)\end{array}$ \\
\hline Face brick & $\begin{array}{c}0.092 \\
(0.302)\end{array}$ & $\begin{array}{c}0.879 \\
(0.508) \\
\end{array}$ & $\begin{array}{l}1922 \\
(120)\end{array}$ & $\begin{array}{l}0.921 \\
(0.22)\end{array}$ & $\begin{array}{c}0.10 \\
(0.59)\end{array}$ \\
\hline Vertical wall air layer & & & & & $\begin{array}{c}0.16 \\
(0.89)\end{array}$ \\
\hline Gypsum board & $\begin{array}{c}0.0127 \\
(0.0417) \\
\end{array}$ & $\begin{array}{c}0.160 \\
(0.0926) \\
\end{array}$ & $\begin{array}{l}800 \\
(50) \\
\end{array}$ & $\begin{array}{l}0.837 \\
(0.2) \\
\end{array}$ & $\begin{array}{l}0.079 \\
(0.45) \\
\end{array}$ \\
\hline Steel studs w/mineral wool insulation, R13 & $\begin{array}{c}0.089 \\
(0.292) \\
\end{array}$ & $\begin{array}{c}0.0751 \\
(0.0434)\end{array}$ & $\begin{array}{l}288 \\
(18)\end{array}$ & $\begin{array}{l}1.298 \\
(0.31) \\
\end{array}$ & $\begin{array}{c}1.2 \\
(6.7)\end{array}$ \\
\hline Gypsum board & $\begin{array}{c}0.0159 \\
(0.0521) \\
\end{array}$ & $\begin{array}{c}0.160 \\
(0.0926)\end{array}$ & $\begin{array}{l}800 \\
(50) \\
\end{array}$ & $\begin{array}{l}0.837 \\
(0.2) \\
\end{array}$ & $\begin{array}{l}0.099 \\
(0.56)\end{array}$ \\
\hline
\end{tabular}




\begin{tabular}{|c|c|c|c|c|c|}
\hline Bismarck and Minneapolis: & Thickness & Conductivity & Density & Specific Heat & Resistance \\
\hline Description & $\begin{array}{c}\mathrm{m} \\
(\mathrm{ft})\end{array}$ & $\begin{array}{c}\mathrm{W} / \mathrm{m}-\mathrm{K} \\
(\mathrm{Btu} / \mathrm{hr}-\mathrm{ft}-\mathrm{F})\end{array}$ & $\begin{array}{l}\mathrm{kg} / \mathrm{m}^{3} \\
\left(\mathrm{lb} / \mathrm{ft}^{3}\right)\end{array}$ & $\begin{array}{c}\mathrm{kJ} / \mathrm{kg}-\mathrm{K} \\
\text { (Btu/lb-F) }\end{array}$ & $\begin{array}{c}\mathrm{m}^{2}-\mathrm{K} / \mathrm{W} \\
\left(\mathrm{hr}-\mathrm{ft}^{2}-\mathrm{F} / \mathrm{Btu}\right)\end{array}$ \\
\hline Face brick & $\begin{array}{c}0.092 \\
(0.302) \\
\end{array}$ & $\begin{array}{c}0.879 \\
(0.508) \\
\end{array}$ & $\begin{array}{l}1922 \\
(120) \\
\end{array}$ & $\begin{array}{l}0.921 \\
(0.22) \\
\end{array}$ & $\begin{array}{c}0.10 \\
(0.59) \\
\end{array}$ \\
\hline Vertical wall air layer & & & & & $\begin{array}{c}0.16 \\
(0.89) \\
\end{array}$ \\
\hline Gypsum board & $\begin{array}{c}0.0127 \\
(0.0417) \\
\end{array}$ & $\begin{array}{c}0.160 \\
(0.0926) \\
\end{array}$ & $\begin{array}{l}800 \\
(50) \\
\end{array}$ & $\begin{array}{c}0.837 \\
(0.2) \\
\end{array}$ & $\begin{array}{l}0.079 \\
(0.45) \\
\end{array}$ \\
\hline Steel studs w/mineral wool/fiber batt R13 & $\begin{array}{c}0.089 \\
(0.292)\end{array}$ & $\begin{array}{c}0.0751 \\
(0.0434) \\
\end{array}$ & $\begin{array}{l}288 \\
(18)\end{array}$ & $\begin{array}{l}1.298 \\
(0.31)\end{array}$ & $\begin{array}{c}1.2 \\
(6.7)\end{array}$ \\
\hline Expanded polystyrene, extruded & $\begin{array}{c}0.0191 \\
(0.0625)\end{array}$ & $\begin{array}{l}0.0277 \\
(0.016)\end{array}$ & $\begin{array}{c}29 \\
(1.8)\end{array}$ & $\begin{array}{l}1.214 \\
(0.29)\end{array}$ & $\begin{array}{l}0.70 \\
(4.0)\end{array}$ \\
\hline Gypsum board & $\begin{array}{c}0.0159 \\
(0.0521)\end{array}$ & $\begin{array}{c}0.160 \\
(0.0926) \\
\end{array}$ & $\begin{array}{l}800 \\
(50) \\
\end{array}$ & $\begin{array}{l}0.837 \\
(0.2) \\
\end{array}$ & $\begin{array}{l}0.099 \\
(0.56) \\
\end{array}$ \\
\hline
\end{tabular}

Masonry Wall Construction:

\begin{tabular}{|c|c|c|c|c|c|}
\hline St. Louis & Thickness & Conductivity & Density & Specific Heat & Resistance \\
\hline Description & $\begin{array}{c}\mathrm{m} \\
(\mathrm{ft})\end{array}$ & $\begin{array}{c}\mathrm{W} / \mathrm{m}-\mathrm{K} \\
(\mathrm{Btu} / \mathrm{hr}-\mathrm{ft}-\mathrm{F})\end{array}$ & $\begin{array}{l}\mathrm{kg} / \mathrm{m}^{3} \\
\left(\mathrm{lb} / \mathrm{ft}^{3}\right)\end{array}$ & $\begin{array}{c}\mathrm{kJ} / \mathrm{kg}-\mathrm{K} \\
\text { (Btu/lb-F) }\end{array}$ & $\begin{array}{c}\mathrm{m}^{2}-\mathrm{K} / \mathrm{W} \\
\left(\mathrm{hr}-\mathrm{ft}^{2}-\mathrm{F} / \mathrm{Btu}\right)\end{array}$ \\
\hline Face brick & $\begin{array}{c}0.092 \\
(0.302) \\
\end{array}$ & $\begin{array}{c}0.879 \\
(0.508) \\
\end{array}$ & $\begin{array}{l}1922 \\
(120) \\
\end{array}$ & $\begin{array}{l}0.921 \\
(0.22) \\
\end{array}$ & $\begin{array}{c}0.10 \\
(0.59) \\
\end{array}$ \\
\hline Vertical wall air layer & & & & & $\begin{array}{c}0.16 \\
(0.89) \\
\end{array}$ \\
\hline Hollow medium weight concrete block & $\begin{array}{c}0.203 \\
(0.667) \\
\end{array}$ & $\begin{array}{c}0.779 \\
(0.450) \\
\end{array}$ & $\begin{array}{l}1842 \\
(115)\end{array}$ & $\begin{array}{l}0.837 \\
(0.2)\end{array}$ & $\begin{array}{c}0.26 \\
(1.48)\end{array}$ \\
\hline Expanded polystyrene, extruded & $\begin{array}{c}0.0254 \\
(0.0833) \\
\end{array}$ & $\begin{array}{l}0.0277 \\
(0.016)\end{array}$ & $\begin{array}{c}29 \\
(1.8)\end{array}$ & $\begin{array}{l}1.214 \\
(0.29)\end{array}$ & $\begin{array}{l}0.88 \\
(5.0) \\
\end{array}$ \\
\hline Gypsum board & $\begin{array}{c}0.0159 \\
(0.0521)\end{array}$ & $\begin{array}{c}0.160 \\
(0.0926)\end{array}$ & $\begin{array}{l}800 \\
(50)\end{array}$ & $\begin{array}{l}0.837 \\
(0.2)\end{array}$ & $\begin{array}{l}0.099 \\
(0.56)\end{array}$ \\
\hline
\end{tabular}

\begin{tabular}{|c|c|c|c|c|c|}
\hline Bismarck and Minneapolis & Thickness & Conductivity & Density & Specific Heat & Resistance \\
\hline Description & $\begin{array}{l}\mathrm{m} \\
(\mathrm{ft})\end{array}$ & $\begin{array}{c}\mathrm{W} / \mathrm{m}-\mathrm{K} \\
\text { (Btu/hr-ft-F) }\end{array}$ & $\begin{array}{l}\mathrm{kg} / \mathrm{m}^{3} \\
\left(\mathrm{lb} / \mathrm{ft}^{3}\right)\end{array}$ & $\begin{array}{c}\text { kJ/kg-K } \\
\text { (Btu/lb-F) }\end{array}$ & $\begin{array}{c}\mathrm{m}^{2}-\mathrm{K} / \mathrm{W} \\
\left(\mathrm{hr}-\mathrm{ft}^{2}-\mathrm{F} / \mathrm{Btu}\right)\end{array}$ \\
\hline Face brick & $\begin{array}{c}0.092 \\
(0.302) \\
\end{array}$ & $\begin{array}{c}0.879 \\
(0.508) \\
\end{array}$ & $\begin{array}{l}1922 \\
(120) \\
\end{array}$ & $\begin{array}{l}0.921 \\
(0.22) \\
\end{array}$ & $\begin{array}{c}0.10 \\
(0.59) \\
\end{array}$ \\
\hline Vertical wall air layer & & & & & $\begin{array}{c}0.16 \\
(0.89) \\
\end{array}$ \\
\hline Hollow medium weight concrete block & $\begin{array}{c}0.203 \\
(0.667) \\
\end{array}$ & $\begin{array}{c}0.779 \\
(0.450) \\
\end{array}$ & $\begin{array}{l}1842 \\
(115)\end{array}$ & $\begin{array}{l}0.837 \\
(0.2) \\
\end{array}$ & $\begin{array}{c}0.26 \\
(1.48) \\
\end{array}$ \\
\hline Expanded polystyrene, extruded & $\begin{array}{c}0.0508 \\
(0.1667)\end{array}$ & $\begin{array}{l}0.0277 \\
(0.016)\end{array}$ & $\begin{array}{c}29 \\
(1.8)\end{array}$ & $\begin{array}{l}1.214 \\
(0.29)\end{array}$ & $\begin{array}{c}1.76 \\
(10.0)\end{array}$ \\
\hline Gypsum board & $\begin{array}{c}0.0159 \\
(0.0521)\end{array}$ & $\begin{array}{c}0.160 \\
(0.0926)\end{array}$ & $\begin{array}{l}800 \\
(50)\end{array}$ & $\begin{array}{l}0.837 \\
(0.2)\end{array}$ & $\begin{array}{l}0.099 \\
(0.56)\end{array}$ \\
\hline
\end{tabular}




\begin{tabular}{|l|c|c|c|c|c|}
\cline { 2 - 6 } \multicolumn{1}{c|}{ Miami and Phoenix } & Thickness & Conductivity & Density & Specific Heat & Resistance \\
\hline Description & $\begin{array}{c}\mathrm{m} \\
(\mathrm{ft})\end{array}$ & $\begin{array}{c}\mathrm{W} / \mathrm{m}-\mathrm{K} \\
(\mathrm{Btu} / \mathrm{hr}-\mathrm{ft}-\mathrm{F})\end{array}$ & $\begin{array}{c}\mathrm{kg} / \mathrm{m}^{3} \\
\left(\mathrm{lb} / \mathrm{ft}^{3}\right)\end{array}$ & $\begin{array}{c}\mathrm{kJ} / \mathrm{kg}-\mathrm{K} \\
(\mathrm{Btu} / \mathrm{lb}-\mathrm{F})\end{array}$ & $\begin{array}{c}\mathrm{m}^{2}-\mathrm{K} / \mathrm{W} \\
\left(\mathrm{hr}-\mathrm{ft}^{2}-\mathrm{F} / \mathrm{Btu}\right)\end{array}$ \\
\hline Face brick & $\begin{array}{c}0.092 \\
(0.302)\end{array}$ & $\begin{array}{c}0.879 \\
(0.508)\end{array}$ & $\begin{array}{c}1922 \\
(120)\end{array}$ & $\begin{array}{c}0.921 \\
(0.22)\end{array}$ & $\begin{array}{c}0.10 \\
(0.59)\end{array}$ \\
\hline Vertical wall air layer & & & & & 0.16 \\
& & & & & $(0.89)$ \\
\hline & 0.203 & 0.779 & 1842 & 0.837 & 0.26 \\
Hollow medium weight concrete block & $(0.667)$ & $(0.450)$ & $(115)$ & $(0.2)$ & $(1.48)$ \\
\hline Gypsum board & 0.0159 & 0.160 & 800 & 0.837 & 0.099 \\
& $(0.0521)$ & $(0.0926)$ & $(50)$ & $(0.2)$ & $(0.56)$ \\
\hline
\end{tabular}

Roof Construction:

\begin{tabular}{|l|c|c|c|c|c|}
\cline { 2 - 6 } \multicolumn{1}{c|}{ All Locations } & Thickness & Conductivity & Density & Specific Heat & Resistance \\
\hline Description & $\begin{array}{c}\mathrm{m} \\
(\mathrm{ft})\end{array}$ & $\begin{array}{c}\mathrm{W} / \mathrm{m}-\mathrm{K} \\
(\mathrm{Btu} / \mathrm{hr}-\mathrm{ft}-\mathrm{F})\end{array}$ & $\begin{array}{c}\mathrm{kg} / \mathrm{m}^{3} \\
\left(\mathrm{lb} / \mathrm{ft}^{3}\right)\end{array}$ & $\begin{array}{c}\mathrm{kJ} / \mathrm{kg}-\mathrm{K} \\
(\mathrm{Btu} / \mathrm{b}-\mathrm{F})\end{array}$ & $\begin{array}{c}\mathrm{m}^{2}-\mathrm{K} / \mathrm{W} \\
\left(\mathrm{hr}-\mathrm{ft}^{2}-\mathrm{F} / \mathrm{Btu}\right)\end{array}$ \\
\hline Built-up roofing & 0.0095 & 1.63 & 1120 & 1.47 & 0.058 \\
& $(0.0313)$ & $(0.939)$ & $(70)$ & $(0.35)$ & $(0.33)$ \\
\hline Polyisocyanurate insulation & 0.0634 & 0.0242 & 24 & 1.59 & 2.62 \\
& $(0.208)$ & $(0.014)$ & $(1.5)$ & $(0.38)$ & $(14.85)$ \\
\hline & 0.0128 & 0.0554 & 288 & 1.298 & 0.23 \\
Vegetable Fiber Board Sheathing & $(0.042)$ & $(0.032)$ & $(18)$ & $(0.31)$ & $(1.32)$ \\
\hline
\end{tabular}

Slab Construction:

\begin{tabular}{|c|c|c|c|c|c|}
\hline All Locations & Thickness & Conductivity & Density & Specific Heat & Resistance \\
\hline Description & $\begin{array}{c}\mathrm{m} \\
(\mathrm{ft})\end{array}$ & $\begin{array}{c}\mathrm{W} / \mathrm{m}-\mathrm{K} \\
\text { (Btu/hr-ft-F) }\end{array}$ & $\begin{array}{l}\mathrm{kg} / \mathrm{m}^{3} \\
\left(\mathrm{lb} / \mathrm{ft}^{3}\right)\end{array}$ & $\begin{array}{c}\mathrm{kJ} / \mathrm{kg}-\mathrm{K} \\
(\mathrm{Btu} / \mathrm{lb}-\mathrm{F})\end{array}$ & $\begin{array}{c}\mathrm{m}^{2}-\mathrm{K} / \mathrm{W} \\
\left(\mathrm{hr}-\mathrm{ft}^{2}-\mathrm{F} / \mathrm{Btu}\right)\end{array}$ \\
\hline Concrete normal weight & $\begin{array}{c}0.127 \\
(0.4167)\end{array}$ & $\begin{array}{c}1.31 \\
(0.7576)\end{array}$ & $\begin{array}{l}2240 \\
(140)\end{array}$ & $\begin{array}{l}0.837 \\
(0.2)\end{array}$ & $\begin{array}{l}0.097 \\
(0.55)\end{array}$ \\
\hline
\end{tabular}

Window:

\begin{tabular}{|l|c|c|}
\cline { 2 - 3 } \multicolumn{1}{c|}{} & U-value & SHGC \\
\hline Location & $\begin{array}{r}\mathrm{W} / \mathrm{m}^{2}-\mathrm{K} \\
\left(\mathrm{Btu} / \mathrm{hr}^{-} \mathrm{ft}^{2}-\mathrm{F}\right)\end{array}$ & \\
\hline St. Louis, Bismarck, and Minneapolis & $\begin{array}{c}.24 \\
(0.57)\end{array}$ & 0.39 \\
\hline Miami and Phoenix & $\begin{array}{c}6.93 \\
(1.22)\end{array}$ & 0.25 \\
\hline
\end{tabular}

The internal gains for the occupied spaces are divided into three parts: lighting, receptacle loads, and occupants. These gains are all applied using a peak value and fraction of peak schedule. The lighting peak is $10.8 \mathrm{~W} / \mathrm{m}^{2}\left(1.0 \mathrm{~W} / \mathrm{ft}^{2}\right)$, the peak receptacle load is $6.8 \mathrm{~W} / \mathrm{m}^{2}\left(0.63 \mathrm{~W} / \mathrm{ft}^{2}\right)$, and the peak occupant density is 53.4 persons $/ 1000 \mathrm{~m}^{2}\left(5\right.$ persons $\left./ 1000 \mathrm{ft}^{2}\right)$. The fraction of peak schedules are shown in Figures 2 to 4 .

The thermostats operate on a setpoint with setback/setup basis. The heating setpoint is $21.1^{\circ} \mathrm{C}\left(70{ }^{\circ} \mathrm{F}\right)$ with a setback temperature of $12.8^{\circ} \mathrm{C}\left(55^{\circ} \mathrm{F}\right)$ and the cooling setpoint is $23.9^{\circ} \mathrm{C}\left(75^{\circ} \mathrm{F}\right)$ with a setup temperature of $32.2^{\circ} \mathrm{C}\left(90^{\circ} \mathrm{F}\right)$. The schedule for the setback/setup differs between weekdays (hours from 6 to 20 at setpoint), Saturdays (hours from 7 to 14 at setpoint) and Sundays (always at setup/setback).

However, for the first hour of operation at setpoint, the system does not bring any outdoor air into the zone. This hour is prior to building occupancy and is used to bring the zone back to setpoint from the setup/setback temperature. 


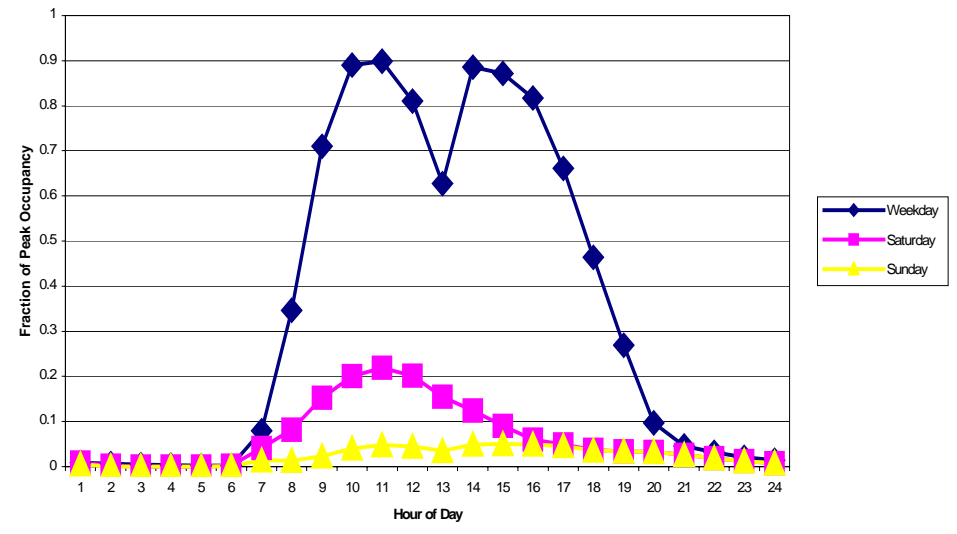

Figure 2 Fractional occupancy schedule for office

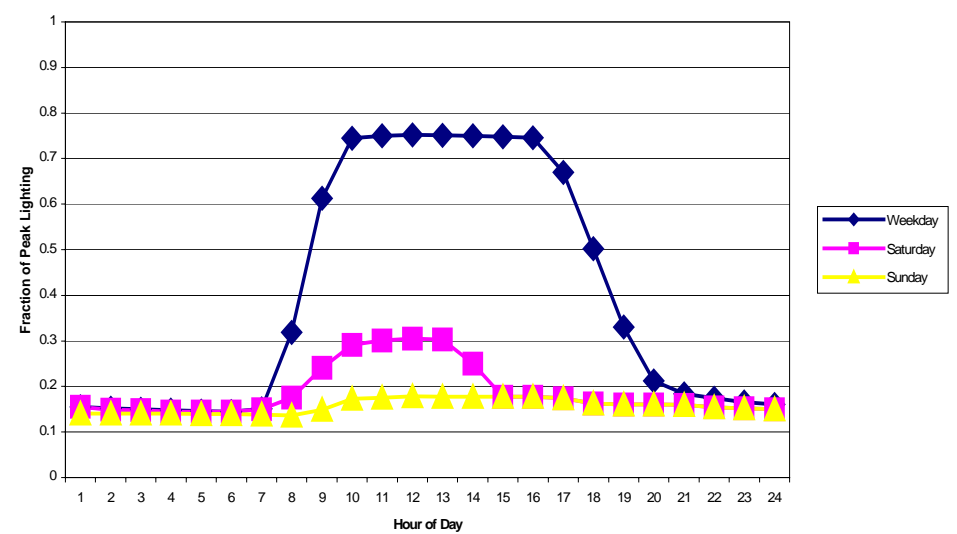

Figure 3 Fractional lighting schedule for office

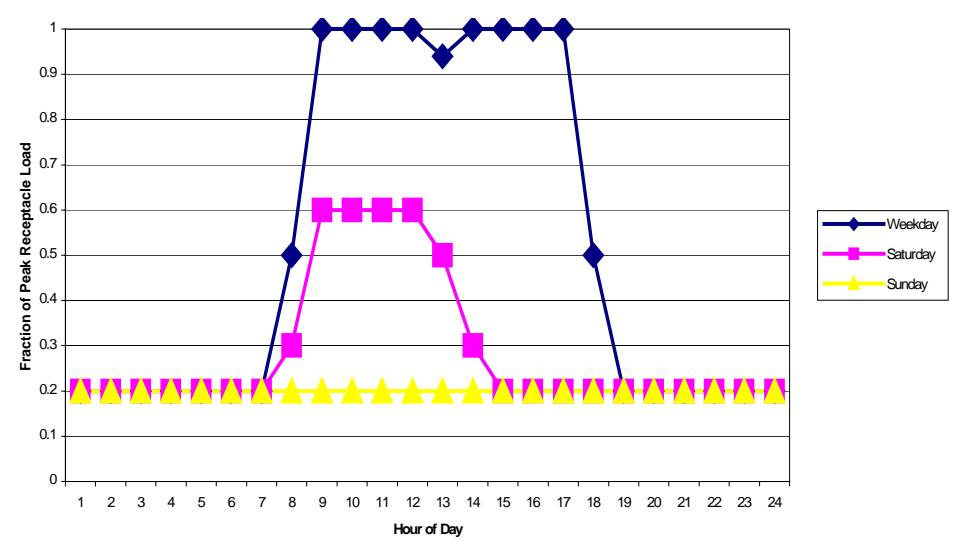

Figure 4 Fractional receptacle load schedule for office 


\section{Retail Building}

The retail building modeled in this study is a one-story building with a total floor area of $1125 \mathrm{~m}^{2}(12,100$ $\mathrm{ft}^{2}$ ) and a floorplan as shown in Figure 5. The building has a window-to-wall ratio of 0.1 with a floor-to-

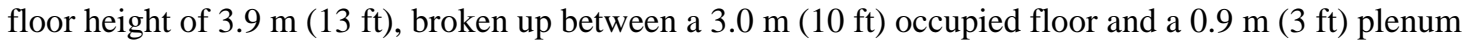
per floor.
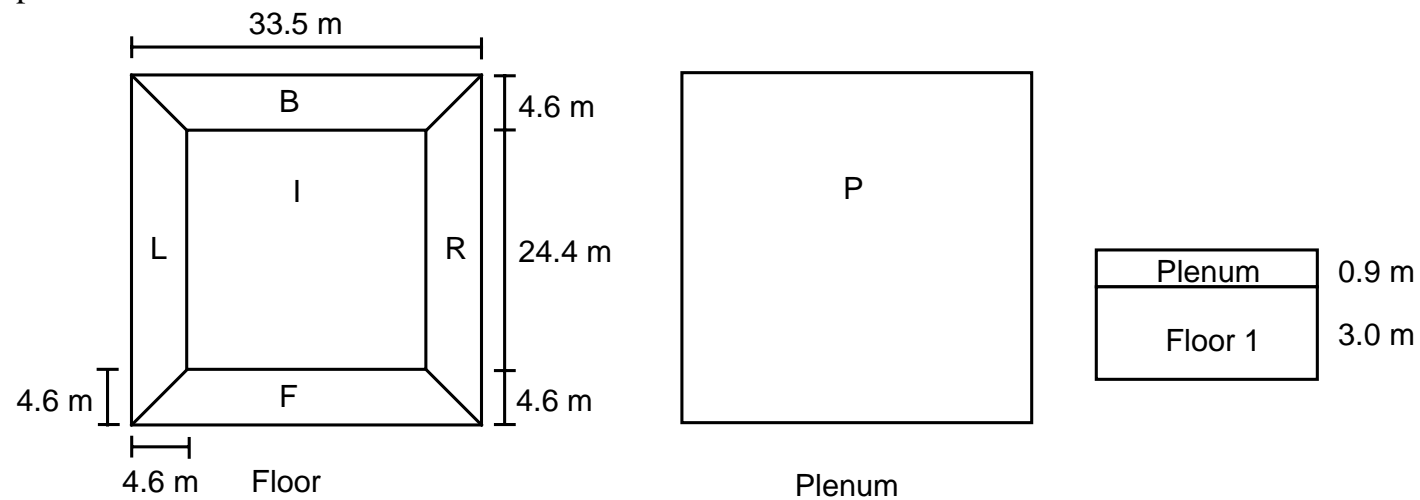

$F, R, B, L$ are the front, right, back, and left perimeter zones.

$I$ is the core zone.

Figure 5 Floorplan of one-story retail building

The wall, roof, slab and window thermal properties used in modeling the retail building are summarized in Table 2.

Table 2 Wall, roof, slab and window thermal properties for retail building

Frame Wall Construction:

\begin{tabular}{l}
\cline { 2 - 7 } \multicolumn{1}{c|}{ St. Louis, Miami and Phoenix } \\
\cline { 2 - 6 }
\end{tabular}




\begin{tabular}{|l|c|c|c|c|c|}
\cline { 2 - 6 } \multicolumn{1}{c|}{ Bismarck and Minneapolis } & Thickness & Conductivity & Density & Specific Heat & Resistance \\
\hline Description & $\begin{array}{c}\mathrm{m} \\
(\mathrm{ft})\end{array}$ & $\begin{array}{c}\mathrm{W} / \mathrm{m}-\mathrm{K} \\
(\mathrm{Btu} / \mathrm{hr}-\mathrm{ft}-\mathrm{F})\end{array}$ & $\begin{array}{c}\mathrm{kg} / \mathrm{m}^{3} \\
\left(\mathrm{lb} / \mathrm{ft}^{3}\right)\end{array}$ & $\begin{array}{c}\mathrm{kJ} / \mathrm{kg}-\mathrm{K} \\
(\mathrm{Btu} / \mathrm{lb}-\mathrm{F})\end{array}$ & $\begin{array}{c}\mathrm{m}^{2}-\mathrm{K} / \mathrm{W} \\
\left(\mathrm{hr}-\mathrm{ft}^{2}-\mathrm{F} / \mathrm{Btu}\right)\end{array}$ \\
\hline Face brick & $\begin{array}{c}0.092 \\
(0.302)\end{array}$ & $\begin{array}{c}0.879 \\
(0.508)\end{array}$ & $\begin{array}{c}1922 \\
(120)\end{array}$ & $\begin{array}{c}0.921 \\
(0.22)\end{array}$ & $\begin{array}{c}0.10 \\
(0.59)\end{array}$ \\
\hline Vertical wall air layer & & & & & 0.16 \\
& & & & & $(0.89)$ \\
\hline Gypsum board & 0.0127 & 0.160 & 800 & 0.837 & 0.079 \\
& $(0.0417)$ & $(0.0926)$ & $(50)$ & $(0.2)$ & $(0.45)$ \\
\hline & 0.089 & 0.0627 & 9.6 & 0.837 & 1.42 \\
Steel studs w/mineral wool/fiber batt R-13 & $(0.292)$ & $(0.0362)$ & $(0.6)$ & $(0.2)$ & $(8.06)$ \\
\hline & 0.0191 & 0.0277 & 29 & 1.214 & 0.70 \\
Expanded polystyrene, extruded & $(0.0625)$ & $(0.016)$ & $(1.8)$ & $(0.29)$ & $(4.0)$ \\
\hline Gypsum board & 0.0159 & 0.160 & 800 & 0.837 & 0.099 \\
& $(0.0521)$ & $(0.0926)$ & $(50)$ & $(0.2)$ & $(0.56)$ \\
\hline
\end{tabular}

Masonry Wall Construction:

\begin{tabular}{|c|c|c|c|c|c|}
\hline St. Louis & Thickness & Conductivity & Density & Specific Heat & Resistance \\
\hline Description & $\begin{array}{c}\mathrm{m} \\
(\mathrm{ft})\end{array}$ & $\begin{array}{c}\mathrm{W} / \mathrm{m}-\mathrm{K} \\
(\mathrm{Btu} / \mathrm{hr}-\mathrm{ft}-\mathrm{F})\end{array}$ & $\begin{array}{l}\mathrm{kg} / \mathrm{m}^{3} \\
\left(\mathrm{lb} / \mathrm{ft}^{3}\right)\end{array}$ & $\begin{array}{c}\mathrm{kJ} / \mathrm{kg}-\mathrm{K} \\
\text { (Btu/lb-F) }\end{array}$ & $\begin{array}{c}\mathrm{m}^{2}-\mathrm{K} / \mathrm{W} \\
\left(\mathrm{hr}-\mathrm{ft}^{2}-\mathrm{F} / \mathrm{Btu}\right)\end{array}$ \\
\hline Face brick & $\begin{array}{c}0.092 \\
(0.302) \\
\end{array}$ & $\begin{array}{c}0.879 \\
(0.508) \\
\end{array}$ & $\begin{array}{l}1922 \\
(120) \\
\end{array}$ & $\begin{array}{l}0.921 \\
(0.22) \\
\end{array}$ & $\begin{array}{c}0.10 \\
(0.59) \\
\end{array}$ \\
\hline Vertical wall air layer & & & & & $\begin{array}{c}0.16 \\
(0.89) \\
\end{array}$ \\
\hline Hollow medium weight concrete block & $\begin{array}{c}0.203 \\
(0.667) \\
\end{array}$ & $\begin{array}{c}0.779 \\
(0.450) \\
\end{array}$ & $\begin{array}{l}1842 \\
(115)\end{array}$ & $\begin{array}{l}0.837 \\
(0.2)\end{array}$ & $\begin{array}{c}0.26 \\
(1.48)\end{array}$ \\
\hline Expanded polystyrene, extruded & $\begin{array}{c}0.0254 \\
(0.0833) \\
\end{array}$ & $\begin{array}{l}0.0277 \\
(0.016)\end{array}$ & $\begin{array}{c}29 \\
(1.8)\end{array}$ & $\begin{array}{l}1.214 \\
(0.29)\end{array}$ & $\begin{array}{l}0.88 \\
(5.0) \\
\end{array}$ \\
\hline Gypsum board & $\begin{array}{c}0.0159 \\
(0.0521)\end{array}$ & $\begin{array}{c}0.160 \\
(0.0926)\end{array}$ & $\begin{array}{l}800 \\
(50)\end{array}$ & $\begin{array}{l}0.837 \\
(0.2)\end{array}$ & $\begin{array}{l}0.099 \\
(0.56)\end{array}$ \\
\hline
\end{tabular}

\begin{tabular}{|c|c|c|c|c|c|}
\hline Bismarck and Minneapolis & Thickness & Conductivity & Density & Specific Heat & Resistance \\
\hline Description & $\begin{array}{l}\mathrm{m} \\
(\mathrm{ft})\end{array}$ & $\begin{array}{c}\mathrm{W} / \mathrm{m}-\mathrm{K} \\
\text { (Btu/hr-ft-F) }\end{array}$ & $\begin{array}{l}\mathrm{kg} / \mathrm{m}^{3} \\
\left(\mathrm{lb} / \mathrm{ft}^{3}\right)\end{array}$ & $\begin{array}{c}\text { kJ/kg-K } \\
\text { (Btu/lb-F) }\end{array}$ & $\begin{array}{c}\mathrm{m}^{2}-\mathrm{K} / \mathrm{W} \\
\left(\mathrm{hr}-\mathrm{ft}^{2}-\mathrm{F} / \mathrm{Btu}\right)\end{array}$ \\
\hline Face brick & $\begin{array}{c}0.092 \\
(0.302) \\
\end{array}$ & $\begin{array}{c}0.879 \\
(0.508) \\
\end{array}$ & $\begin{array}{l}1922 \\
(120) \\
\end{array}$ & $\begin{array}{l}0.921 \\
(0.22) \\
\end{array}$ & $\begin{array}{c}0.10 \\
(0.59) \\
\end{array}$ \\
\hline Vertical wall air layer & & & & & $\begin{array}{c}0.16 \\
(0.89) \\
\end{array}$ \\
\hline Hollow medium weight concrete block & $\begin{array}{c}0.203 \\
(0.667) \\
\end{array}$ & $\begin{array}{c}0.779 \\
(0.450) \\
\end{array}$ & $\begin{array}{l}1842 \\
(115)\end{array}$ & $\begin{array}{l}0.837 \\
(0.2) \\
\end{array}$ & $\begin{array}{c}0.26 \\
(1.48) \\
\end{array}$ \\
\hline Expanded polystyrene, extruded & $\begin{array}{c}0.0508 \\
(0.1667)\end{array}$ & $\begin{array}{l}0.0277 \\
(0.016)\end{array}$ & $\begin{array}{c}29 \\
(1.8)\end{array}$ & $\begin{array}{l}1.214 \\
(0.29)\end{array}$ & $\begin{array}{c}1.76 \\
(10.0)\end{array}$ \\
\hline Gypsum board & $\begin{array}{c}0.0159 \\
(0.0521)\end{array}$ & $\begin{array}{c}0.160 \\
(0.0926)\end{array}$ & $\begin{array}{l}800 \\
(50)\end{array}$ & $\begin{array}{l}0.837 \\
(0.2)\end{array}$ & $\begin{array}{l}0.099 \\
(0.56)\end{array}$ \\
\hline
\end{tabular}




\begin{tabular}{|l|c|c|c|c|c|}
\cline { 2 - 6 } \multicolumn{1}{c|}{ Miami and Phoenix } & Thickness & Conductivity & Density & Specific Heat & Resistance \\
\hline Description & $\begin{array}{c}\mathrm{m} \\
(\mathrm{ft})\end{array}$ & $\begin{array}{c}\mathrm{W} / \mathrm{m}-\mathrm{K} \\
(\mathrm{Btu} / \mathrm{hr}-\mathrm{ft}-\mathrm{F})\end{array}$ & $\begin{array}{c}\mathrm{kg} / \mathrm{m}^{3} \\
\left(\mathrm{lb} / \mathrm{ft}^{3}\right)\end{array}$ & $\begin{array}{c}\mathrm{kJ} / \mathrm{kg}-\mathrm{K} \\
(\mathrm{Btu} / \mathrm{lb}-\mathrm{F})\end{array}$ & $\begin{array}{c}\mathrm{m}^{2}-\mathrm{K} / \mathrm{W} \\
\left(\mathrm{hr}-\mathrm{ft}^{2}-\mathrm{F} / \mathrm{Btu}\right)\end{array}$ \\
\hline Face brick & $\begin{array}{c}0.092 \\
(0.302)\end{array}$ & $\begin{array}{c}0.879 \\
(0.508)\end{array}$ & $\begin{array}{c}1922 \\
(120)\end{array}$ & $\begin{array}{c}0.921 \\
(0.22)\end{array}$ & $\begin{array}{c}0.10 \\
(0.59)\end{array}$ \\
\hline Vertical wall air layer & & & & & 0.16 \\
& & & & & $(0.89)$ \\
\hline & 0.203 & 0.779 & 1842 & 0.837 & 0.26 \\
Hollow medium weight concrete block & $(0.667)$ & $(0.450)$ & $(115)$ & $(0.2)$ & $(1.48)$ \\
\hline Gypsum board & 0.0159 & 0.160 & 800 & 0.837 & 0.099 \\
& $(0.0521)$ & $(0.0926)$ & $(50)$ & $(0.2)$ & $(0.56)$ \\
\hline
\end{tabular}

Roof Construction:

\begin{tabular}{|l|c|c|c|c|c|}
\cline { 2 - 6 } \multicolumn{1}{c|}{ All Locations } & Thickness & Conductivity & Density & Specific Heat & Resistance \\
\hline Description & $\begin{array}{c}\mathrm{m} \\
(\mathrm{ft})\end{array}$ & $\begin{array}{c}\mathrm{W} / \mathrm{m}-\mathrm{K} \\
(\mathrm{Btu} / \mathrm{hr}-\mathrm{ft}-\mathrm{F})\end{array}$ & $\begin{array}{c}\mathrm{kg} / \mathrm{m}^{3} \\
\left(\mathrm{lb} / \mathrm{ft}^{3}\right)\end{array}$ & $\begin{array}{c}\mathrm{kJ} / \mathrm{kg}-\mathrm{K} \\
(\mathrm{Btu} / \mathrm{lb}-\mathrm{F})\end{array}$ & $\begin{array}{c}\mathrm{m}^{2}-\mathrm{K} / \mathrm{W} \\
\left(\mathrm{hr}^{2}-\mathrm{ft}^{2}-\mathrm{F} / \mathrm{Btu}\right)\end{array}$ \\
\hline Modified bitumen mineral surface roofing & 0.0063 & 0.109 & 1120 & 1.47 & 0.058 \\
& $(0.0208)$ & $(0.063)$ & $(70)$ & $(0.35)$ & $(0.33)$ \\
\hline & 0.0128 & 0.0554 & 288 & 1.298 & 0.23 \\
Vegetable fiber board coverboard & $(0.042)$ & $(0.032)$ & $(18)$ & $(0.31)$ & $(1.32)$ \\
\hline Polyisocyanurate insulation - aged value & 0.0634 & 0.0312 & 24.0 & 1.59 & $\begin{array}{c}2.42 \\
(13.75)\end{array}$ \\
\hline
\end{tabular}

Slab Construction:

\begin{tabular}{|c|c|c|c|c|c|}
\hline All Locations & Thickness & Conductivity & Density & Specific Heat & Resistance \\
\hline Description & $\begin{array}{c}\mathrm{m} \\
(\mathrm{ft})\end{array}$ & $\begin{array}{c}\mathrm{W} / \mathrm{m}-\mathrm{K} \\
\text { (Btu/hr-ft-F) }\end{array}$ & $\begin{array}{l}\mathrm{kg} / \mathrm{m}^{3} \\
\left(\mathrm{lb} / \mathrm{ft}^{3}\right)\end{array}$ & $\begin{array}{c}\mathrm{kJ} / \mathrm{kg}-\mathrm{K} \\
(\mathrm{Btu} / \mathrm{lb}-\mathrm{F})\end{array}$ & $\begin{array}{c}\mathrm{m}^{2}-\mathrm{K} / \mathrm{W} \\
\left(\mathrm{hr}-\mathrm{ft}^{2}-\mathrm{F} / \mathrm{Btu}\right)\end{array}$ \\
\hline Concrete normal weight & $\begin{array}{c}0.127 \\
(0.4167)\end{array}$ & $\begin{array}{c}1.31 \\
(0.7576)\end{array}$ & $\begin{array}{l}2240 \\
(140)\end{array}$ & $\begin{array}{l}0.837 \\
(0.2)\end{array}$ & $\begin{array}{l}0.097 \\
(0.55)\end{array}$ \\
\hline
\end{tabular}

Window:

\begin{tabular}{|l|c|c|}
\cline { 2 - 3 } \multicolumn{1}{c|}{} & U-value & SHGC \\
\hline Location & $\begin{array}{c}\mathrm{W} / \mathrm{m}^{2}-\mathrm{K} \\
\left(\mathrm{Btu} / \mathrm{hr}^{-} \mathrm{ft}^{2}-\mathrm{F}\right)\end{array}$ & \\
\hline St. Louis & $\begin{array}{c}3.24 \\
(0.57)\end{array}$ & 0.39 \\
\hline Bismarck and Minneapolis & 3.24 & \\
\hline Miami and Phoenix & $(0.57)$ & 0.49 \\
\hline
\end{tabular}

The internal gains for the occupied spaces are divided into three parts: lighting, receptacle loads, and occupants. These gains are all applied using a peak value and fraction of peak schedule. The lighting peak is $16.15 \mathrm{~W} / \mathrm{m}^{2}\left(1.5 \mathrm{~W} / \mathrm{ft}^{2}\right)$, the peak receptacle load is $2.58 \mathrm{~W} / \mathrm{m}^{2}\left(0.24 \mathrm{~W} / \mathrm{ft}^{2}\right)$, and the peak occupant density is 162 persons $/ 1000 \mathrm{~m}^{2}\left(15\right.$ persons $\left./ 1000 \mathrm{ft}^{2}\right)$. The fraction of peak schedules are shown in Figures 6 to 8.

The thermostats operate on a setpoint with setback/setup basis. The heating setpoint is $21.1^{\circ} \mathrm{C}\left(70{ }^{\circ} \mathrm{F}\right)$ with a setback temperature of $12.8^{\circ} \mathrm{C}\left(55^{\circ} \mathrm{F}\right)$ and the cooling setpoint is $23.9^{\circ} \mathrm{C}\left(75^{\circ} \mathrm{F}\right)$ with a setup temperature of $37.2^{\circ} \mathrm{C}\left(99^{\circ} \mathrm{F}\right)$. The schedule for the setback/setup differs between weekdays (hours from 7 to 21 at setpoint), Saturdays (hours from 7 to 21 at setpoint) and Sundays (hours from 9 to 19 at setpoint). However for the first hour of operation at setpoint the system does not bring any outdoor air into the zone. 
This hour is prior to building occupancy and is used to bring the zone back to setpoint from the setup/setback temperature.

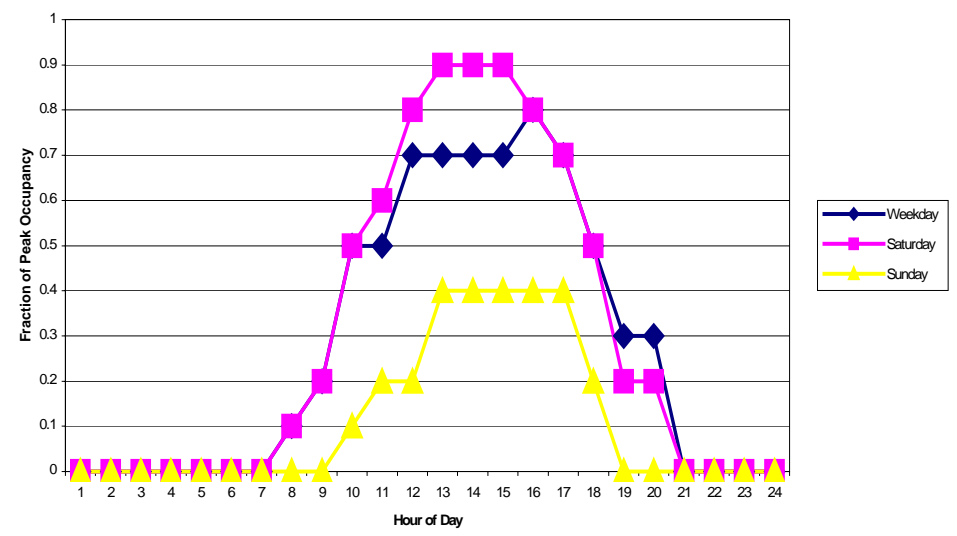

Figure 6 Fractional occupancy schedule for retail

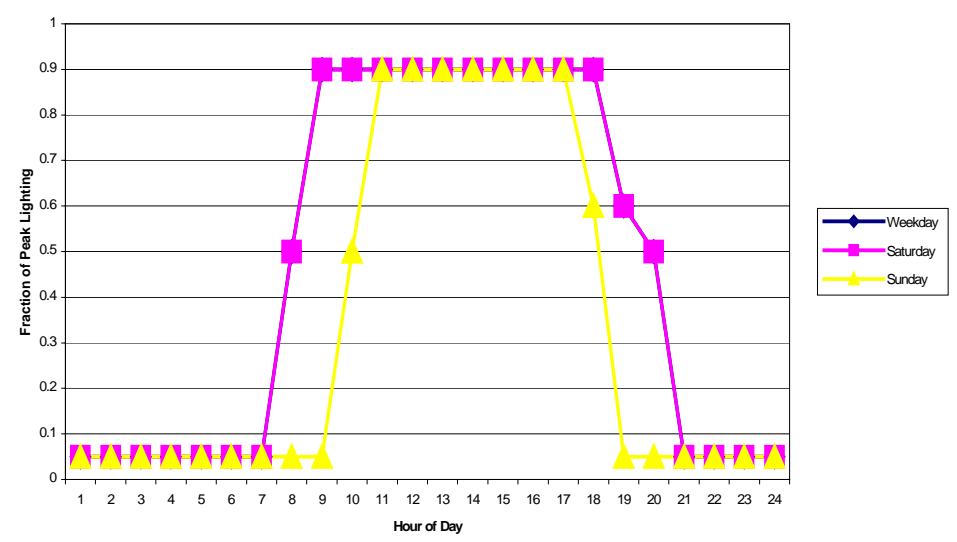

Figure 7 Fractional lighting schedule for retail

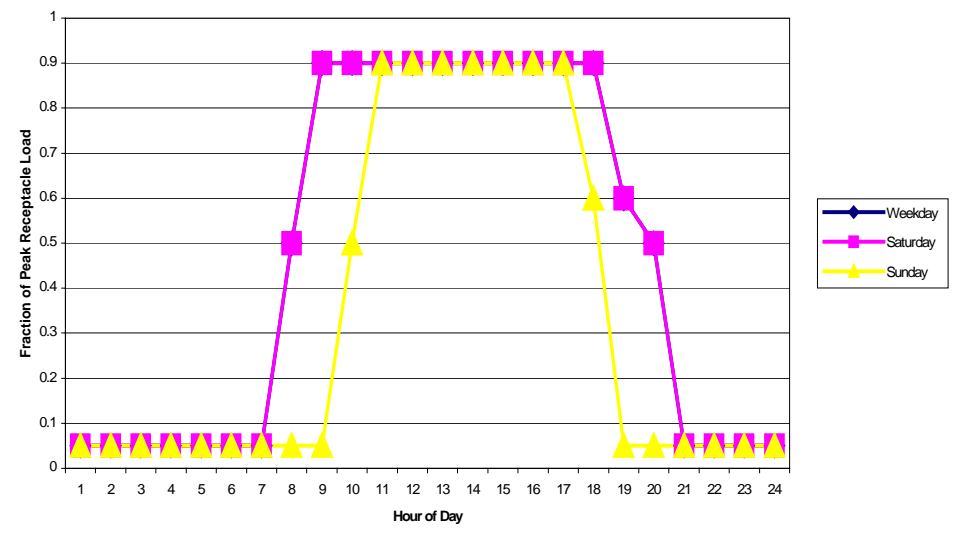

Figure 8 Fractional receptacle load schedule for retail 


\section{Residential Building}

The residential building modeled in this study is a four story building with a total floor area of $3425 \mathrm{~m}^{2}$ $\left(36,864 \mathrm{ft}^{2}\right)$ and a floorplan as shown in Figure 9. The building has a window-to-wall ratio of 0.25 with a floor-to-floor height of $3.0 \mathrm{~m}(10 \mathrm{ft})$, broken up between a $2.4 \mathrm{~m}(8 \mathrm{ft})$ occupied floor and a $0.6 \mathrm{~m}(2 \mathrm{ft})$ plenum for the bottom three floors and a $2.4 \mathrm{~m}(8 \mathrm{ft})$ occupied floor and a $3.0 \mathrm{~m}(10 \mathrm{ft})$ attic at the top. The attic has a pitched roof and is vented. The building also includes a single elevator shaft with two elevators. The corner units will have a doorway connecting to the corridor. One of bottom mid-units is used as a lobby entrance instead of a living unit.
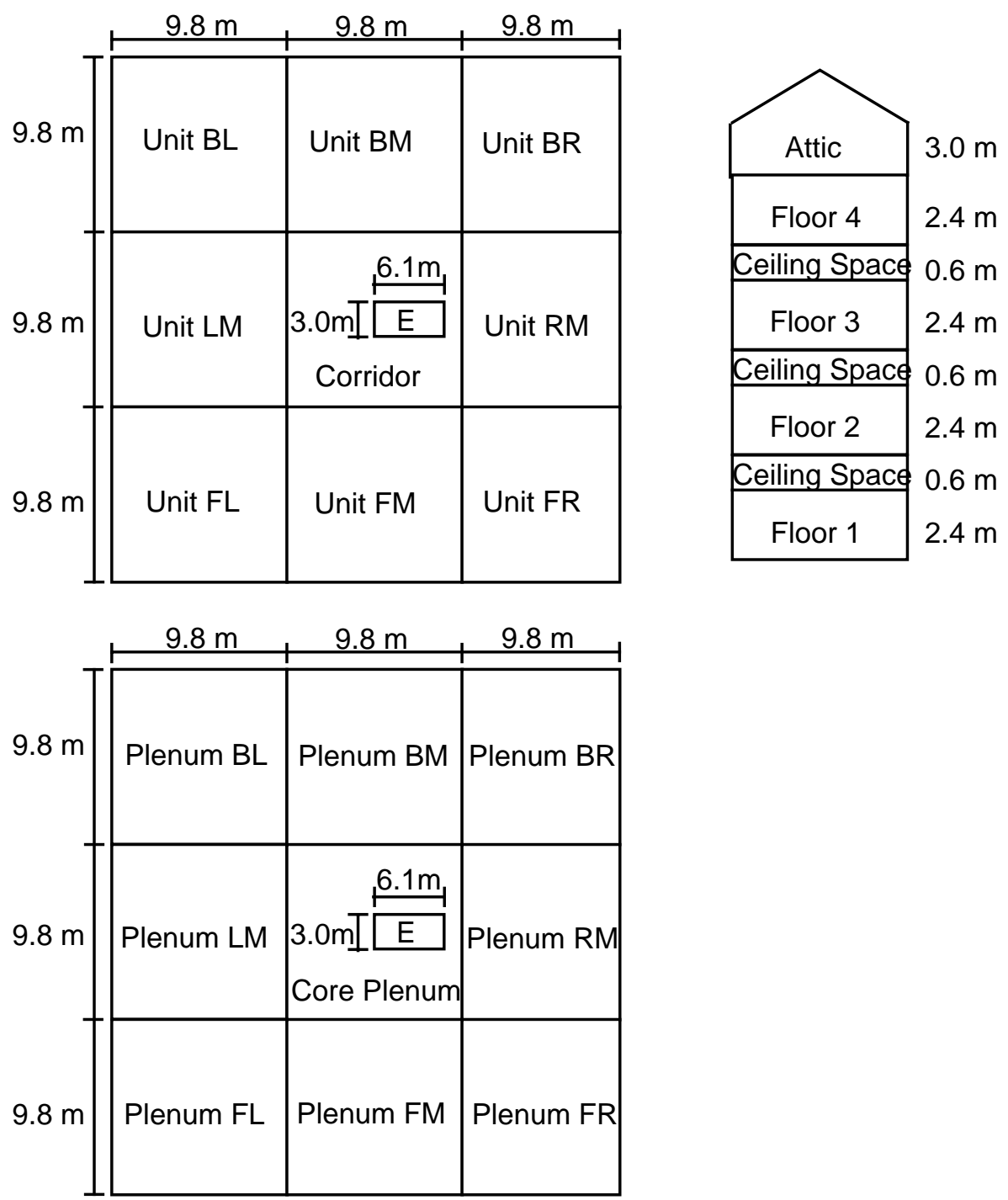

Figure 9 Floorplan of modeled four-story residential building

The wall, roof, slab and window thermal properties used for modeling the apartment building are summarized in Table 3. 
Table 3 Wall, roof, slab and window thermal properties for apartment building

Brick Walls Construction:

Bismarck and Minneapolis

\begin{tabular}{|c|c|c|c|c|c|}
\hline Description & $\begin{array}{c}\mathrm{m} \\
(\mathrm{ft})\end{array}$ & $\begin{array}{c}\mathrm{W} / \mathrm{m}-\mathrm{K} \\
(\mathrm{Btu} / \mathrm{hr}-\mathrm{ft}-\mathrm{F})\end{array}$ & $\begin{array}{l}\mathrm{kg} / \mathrm{m}^{3} \\
\left(\mathrm{lb} / \mathrm{ft}^{3}\right)\end{array}$ & $\begin{array}{l}\mathrm{kJ} / \mathrm{kg}-\mathrm{K} \\
(\mathrm{Btu} / \mathrm{lb}-\mathrm{F})\end{array}$ & $\begin{array}{c}\mathrm{m}^{2}-\mathrm{K} / \mathrm{W} \\
\left(\mathrm{hr}-\mathrm{ft}^{2}-\mathrm{F} / \mathrm{Btu}\right)\end{array}$ \\
\hline Face brick & $\begin{array}{c}0.092 \\
(0.302) \\
\end{array}$ & $\begin{array}{c}0.879 \\
(0.508)\end{array}$ & $\begin{array}{l}1922 \\
(120)\end{array}$ & $\begin{array}{l}0.921 \\
(0.22)\end{array}$ & $\begin{array}{c}0.10 \\
(0.59)\end{array}$ \\
\hline Vertical wall air layer & & & & & $\begin{array}{c}0.16 \\
(0.89)\end{array}$ \\
\hline $\begin{array}{l}\text { Extruded polystyrene foam insulating } \\
\text { sheathing }\end{array}$ & $\begin{array}{c}0.0191 \\
(0.0625) \\
\end{array}$ & $\begin{array}{l}0.0294 \\
(0.017) \\
\end{array}$ & $\begin{array}{l}28.8 \\
(1.8)\end{array}$ & $\begin{array}{c}1.21 \\
(0.29) \\
\end{array}$ & $\begin{array}{c}0.66 \\
(3.75) \\
\end{array}$ \\
\hline OSB sheathing & $\begin{array}{l}0.0127 \\
(0.042)\end{array}$ & $\begin{array}{l}0.0917 \\
(0.053)\end{array}$ & $\begin{array}{l}288 \\
(18)\end{array}$ & $\begin{array}{c}1.30 \\
(0.31)\end{array}$ & $\begin{array}{l}0.139 \\
(0.79)\end{array}$ \\
\hline Wood studs w/ batt R-13 & $\begin{array}{c}0.089 \\
(0.292) \\
\end{array}$ & $\begin{array}{l}0.0485 \\
(0.028) \\
\end{array}$ & $\begin{array}{c}73.4 \\
(4.58) \\
\end{array}$ & $\begin{array}{l}0.837 \\
(0.2) \\
\end{array}$ & $\begin{array}{c}1.82 \\
(10.34) \\
\end{array}$ \\
\hline Gypsum board & $\begin{array}{l}0.0127 \\
(0.042) \\
\end{array}$ & $\begin{array}{c}0.160 \\
(0.0926)\end{array}$ & $\begin{array}{r}800 \\
(50)\end{array}$ & $\begin{array}{r}0.837 \\
(0.2)\end{array}$ & $\begin{array}{l}0.079 \\
(0.45)\end{array}$ \\
\hline
\end{tabular}

St Louis, Miami, Phoenix

\begin{tabular}{|l|c|c|c|c|c|}
\hline Description & $\begin{array}{c}\mathrm{m} \\
(\mathrm{ft})\end{array}$ & $\begin{array}{c}\mathrm{W} / \mathrm{m}-\mathrm{K} \\
(\mathrm{Btu} / \mathrm{hr}-\mathrm{ft}-\mathrm{F})\end{array}$ & $\begin{array}{c}\mathrm{kg} / \mathrm{m}^{3} \\
\left(\mathrm{lb} / \mathrm{ft}^{3}\right)\end{array}$ & $\begin{array}{c}\mathrm{kJ} / \mathrm{kg}-\mathrm{K} \\
(\mathrm{Btu} / \mathrm{lb}-\mathrm{F})\end{array}$ & $\begin{array}{c}\mathrm{m}^{2}-\mathrm{K} / \mathrm{W} \\
\left(\mathrm{hr}-\mathrm{ft}^{2}-\mathrm{F} / \mathrm{Btu}\right)\end{array}$ \\
\hline Face brick & $\begin{array}{c}0.092 \\
(0.302)\end{array}$ & $\begin{array}{c}0.879 \\
(0.508)\end{array}$ & $\begin{array}{c}1922 \\
(120)\end{array}$ & $\begin{array}{c}0.921 \\
(0.22)\end{array}$ & $\begin{array}{c}0.10 \\
(0.59)\end{array}$ \\
\hline Vertical wall air layer & & & & & 0.16 \\
& & & & & $(0.89)$ \\
\hline OSB sheathing & 0.0127 & 0.0917 & 288 & 1.30 & 0.139 \\
& $(0.042)$ & $(0.053)$ & $(18)$ & $(0.31)$ & $(0.79)$ \\
\hline Wood studs w/ batt R-13 & 0.089 & 0.0485 & 73.4 & 0.837 & 1.82 \\
& $(0.292)$ & $(0.028)$ & $(4.58)$ & $(0.2)$ & $(10.34)$ \\
\hline Gypsum board & 0.0127 & 0.160 & 800 & 0.837 & 0.079 \\
& $(0.042)$ & $(0.0926)$ & $(50)$ & $(0.2)$ & $(0.45)$ \\
\hline
\end{tabular}

Clapboard Wall Construction:

\begin{tabular}{|l|c|c|c|c|c|}
\cline { 2 - 6 } \multicolumn{1}{c|}{ Bismarck and Minneapolis } & Thickness & Conductivity & Density & Specific Heat & Resistance \\
\hline Description & $\begin{array}{c}\mathrm{m} \\
(\mathrm{ft})\end{array}$ & $\begin{array}{c}\mathrm{W} / \mathrm{m}-\mathrm{K} \\
(\mathrm{Btu} / \mathrm{hr}-\mathrm{ft}-\mathrm{F})\end{array}$ & $\begin{array}{c}\mathrm{kg} / \mathrm{m}^{3} \\
\left(\mathrm{lb} / \mathrm{ft}^{3}\right)\end{array}$ & $\begin{array}{c}\mathrm{kJ} / \mathrm{kg}-\mathrm{K} \\
(\mathrm{Btu} / \mathrm{lb}-\mathrm{F})\end{array}$ & $\begin{array}{c}\mathrm{m}^{2}-\mathrm{K} / \mathrm{W} \\
\left(\mathrm{hr}^{\mathrm{f}} \mathrm{ft}-\mathrm{F} / \mathrm{Btu}\right)\end{array}$ \\
\hline & 0.0634 & 0.171 & 1922 & 1.00 & 0.037 \\
Wood fiber-cement clapboards & $(0.208)$ & $(0.099)$ & $(120)$ & $(0.24)$ & $(0.21)$ \\
\hline Extruded polystyrene foam insulating & 0.0191 & 0.0294 & 28.8 & 1.21 & 0.66 \\
sheathing & $(0.0625)$ & $(0.017)$ & $(1.8)$ & $(0.29)$ & $(3.75)$ \\
\hline OSB sheathing & 0.0127 & 0.0917 & 288 & 1.30 & 0.139 \\
& $(0.042)$ & $(0.053)$ & $(18)$ & $(0.31)$ & $(0.79)$ \\
\hline Wood studs w/ batt R-13 & 0.089 & 0.0485 & 73.4 & 0.837 & 1.82 \\
& $(0.292)$ & $(0.028)$ & $(4.58)$ & $(0.2)$ & $(10.34)$ \\
\hline Gypsum board & 0.0127 & 0.160 & 800 & 0.837 & 0.079 \\
& $(0.042)$ & $(0.0926)$ & $(50)$ & $(0.2)$ & $(0.45)$ \\
\hline
\end{tabular}




\begin{tabular}{|l|c|c|c|c|c|}
\cline { 2 - 6 } \multicolumn{1}{c|}{ St Louis, Miami, Phoenix } & Thickness & Conductivity & Density & Specific Heat & Resistance \\
\hline Description & $\begin{array}{c}\mathrm{m} \\
(\mathrm{ft})\end{array}$ & $\begin{array}{c}\mathrm{W} / \mathrm{m}-\mathrm{K} \\
(\mathrm{Btu} / \mathrm{hr}-\mathrm{ft}-\mathrm{F})\end{array}$ & $\begin{array}{c}\mathrm{kg} / \mathrm{m}^{3} \\
\left(\mathrm{lb} / \mathrm{ft}^{3}\right)\end{array}$ & $\begin{array}{c}\mathrm{kJ} / \mathrm{kg}-\mathrm{K} \\
(\mathrm{Btu} / \mathrm{lb}-\mathrm{F})\end{array}$ & $\begin{array}{c}\mathrm{m}^{2}-\mathrm{K} / \mathrm{W} \\
\left(\mathrm{hr}-\mathrm{ft}^{2}-\mathrm{F} / \mathrm{Btu}\right)\end{array}$ \\
\hline & 0.0634 & 0.171 & 1922 & 1.00 & 0.037 \\
Wood fiber-cement clapboards & $(0.208)$ & $(0.099)$ & $(120)$ & $(0.24)$ & $(0.21)$ \\
\hline OSB sheathing & 0.0127 & 0.0917 & 288 & 1.30 & 0.139 \\
& $(0.042)$ & $(0.053)$ & $(18)$ & $(0.31)$ & $(0.79)$ \\
\hline Wood studs w/ batt R-13 & 0.089 & 0.0485 & 73.4 & 0.837 & 1.82 \\
& $(0.292)$ & $(0.028)$ & $(4.58)$ & $(0.2)$ & $(10.34)$ \\
\hline Gypsum board & 0.0127 & 0.160 & 800 & 0.837 & 0.079 \\
& $(0.042)$ & $(0.0926)$ & $(50)$ & $(0.2)$ & $(0.45)$ \\
\hline
\end{tabular}

Roof Construction:

\begin{tabular}{|l|c|c|c|c|c|}
\cline { 2 - 6 } \multicolumn{1}{c|}{ All Locations } & Thickness & Conductivity & Density & Specific Heat & Resistance \\
\hline Description & $\begin{array}{c}\mathrm{m} \\
(\mathrm{ft})\end{array}$ & $\begin{array}{c}\mathrm{W} / \mathrm{m}-\mathrm{K} \\
(\mathrm{Btu} / \mathrm{hr}-\mathrm{ft}-\mathrm{F})\end{array}$ & $\begin{array}{c}\mathrm{kg} / \mathrm{m}^{3} \\
\left(\mathrm{lb} / \mathrm{ft}^{3}\right)\end{array}$ & $\begin{array}{c}\mathrm{kJ} / \mathrm{kg}-\mathrm{K} \\
(\mathrm{Btu} / \mathrm{lb}-\mathrm{F})\end{array}$ & $\begin{array}{c}\mathrm{m}^{2}-\mathrm{K} / \mathrm{W} \\
\left(\mathrm{hr}-\mathrm{ft}^{2}-\mathrm{F} / \mathrm{Btu}\right)\end{array}$ \\
\hline Asphalt shingles & $\begin{array}{c}0.0114 \\
\end{array}$ & $\begin{array}{c}1.475 \\
(0.0375)\end{array}$ & $\begin{array}{c}1120 \\
(0.852)\end{array}$ & $\begin{array}{c}1.47 \\
(70)\end{array}$ & $\begin{array}{c}0.078 \\
(0.35)\end{array}$ \\
\hline Oriented strand board (waferboard) & 0.0128 & 0.0917 & 288 & 1.298 & 0.14 \\
sheathing & $(0.042)$ & $(0.053)$ & $(18)$ & $(0.31)$ & $(0.79)$ \\
\hline
\end{tabular}

Top Floor Ceiling Construction:

\begin{tabular}{|c|c|c|c|c|c|}
\hline All Locations & Thickness & Conductivity & Density & Specific Heat & Resistance \\
\hline Description & $\begin{array}{c}\mathrm{m} \\
(\mathrm{ft})\end{array}$ & $\begin{array}{c}\mathrm{W} / \mathrm{m}-\mathrm{K} \\
(\mathrm{Btu} / \mathrm{hr}-\mathrm{ft}-\mathrm{F})\end{array}$ & $\begin{array}{l}\mathrm{kg} / \mathrm{m}^{3} \\
\left(\mathrm{lb} / \mathrm{ft}^{3}\right)\end{array}$ & $\begin{array}{c}\mathrm{kJ} / \mathrm{kg}-\mathrm{K} \\
(\mathrm{Btu} / \mathrm{lb}-\mathrm{F})\end{array}$ & $\begin{array}{c}\mathrm{m}^{2}-\mathrm{K} / \mathrm{W} \\
\left(\mathrm{hr}-\mathrm{ft}^{2}-\mathrm{F} / \mathrm{Btu}\right)\end{array}$ \\
\hline Insulated wood trusses & $\begin{array}{c}0.254 \\
(0.833)\end{array}$ & $\begin{array}{c}0.0389 \\
(0.0225) \\
\end{array}$ & $\begin{array}{c}57.5 \\
(3.59)\end{array}$ & $\begin{array}{l}0.837 \\
(0.2) \\
\end{array}$ & $\begin{array}{c}6.52 \\
(37.04) \\
\end{array}$ \\
\hline
\end{tabular}

Slab Construction:

\begin{tabular}{|c|c|c|c|c|c|}
\hline All Locations & Thickness & Conductivity & Density & Specific Heat & Resistance \\
\hline Description & $\begin{array}{c}\mathrm{m} \\
(\mathrm{ft})\end{array}$ & $\begin{array}{c}\mathrm{W} / \mathrm{m}-\mathrm{K} \\
(\mathrm{Btu} / \mathrm{hr}-\mathrm{ft}-\mathrm{F})\end{array}$ & $\begin{array}{l}\mathrm{kg} / \mathrm{m}^{3} \\
\left(\mathrm{lb} / \mathrm{ft}^{3}\right)\end{array}$ & $\begin{array}{c}\mathrm{kJ} / \mathrm{kg}-\mathrm{K} \\
(\mathrm{Btu} / \mathrm{lb}-\mathrm{F})\end{array}$ & $\begin{array}{c}\mathrm{m}^{2}-\mathrm{K} / \mathrm{W} \\
\left(\mathrm{hr}-\mathrm{ft}^{2}-\mathrm{F} / \mathrm{Btu}\right)\end{array}$ \\
\hline Concrete normal weight & $\begin{array}{c}0.127 \\
(0.4167)\end{array}$ & $\begin{array}{c}1.31 \\
(0.7576)\end{array}$ & $\begin{array}{l}2240 \\
(140)\end{array}$ & $\begin{array}{l}0.837 \\
(0.2) \\
\end{array}$ & $\begin{array}{l}0.097 \\
(0.55)\end{array}$ \\
\hline
\end{tabular}

Floor Construction:

\begin{tabular}{|l|c|c|c|c|c|}
\cline { 2 - 6 } \multicolumn{1}{c|}{ All Locations } & Thickness & Conductivity & Density & Specific Heat & Resistance \\
\hline Description & $\begin{array}{c}\mathrm{m} \\
(\mathrm{ft})\end{array}$ & $\begin{array}{c}\mathrm{W} / \mathrm{m}-\mathrm{K} \\
(\mathrm{Btu} / \mathrm{hr}-\mathrm{ft}-\mathrm{F})\end{array}$ & $\begin{array}{c}\mathrm{kg} / \mathrm{m}^{3} \\
\left(\mathrm{lb} / \mathrm{ft}^{3}\right)\end{array}$ & $\begin{array}{c}\mathrm{kJ} / \mathrm{kg}-\mathrm{K} \\
(\mathrm{Btu} / \mathrm{lb}-\mathrm{F})\end{array}$ & $\begin{array}{c}\mathrm{m}^{2}-\mathrm{K} / \mathrm{W} \\
\left(\mathrm{hr}-\mathrm{ft}^{2}-\mathrm{F} / \mathrm{Btu}\right)\end{array}$ \\
\hline Gypcrete & 0.0128 & 0.160 & 800 & 0.84 & 0.079 \\
& $(0.042)$ & $(0.0926)$ & $(50)$ & $(0.2)$ & $(0.45)$ \\
\hline Plywood & 0.0191 & 0.116 & 545 & 1.21 & 0.164 \\
& $(0.0625)$ & $(0.0669)$ & $(34)$ & $(0.29)$ & $(0.93)$ \\
\hline Wood Joists w/acoustic batts & 0.254 & 0.0673 & 57.5 & 1.67 & 3.78 \\
& $(0.833)$ & $(0.0389)$ & $(3.59)$ & $(0.4)$ & $(21.44)$ \\
\hline
\end{tabular}


Ceiling (except top floor) Construction:

\begin{tabular}{|c|c|c|c|c|c|}
\hline All Locations & Thickness & Conductivity & Density & Specific Heat & Resistance \\
\hline Description & $\begin{array}{c}\mathrm{m} \\
(\mathrm{ft})\end{array}$ & $\begin{array}{c}\mathrm{W} / \mathrm{m}-\mathrm{K} \\
(\mathrm{Btu} / \mathrm{hr}-\mathrm{ft}-\mathrm{F})\end{array}$ & $\begin{array}{l}\mathrm{kg} / \mathrm{m}^{3} \\
\left(\mathrm{lb} / \mathrm{ft}^{3}\right)\end{array}$ & $\begin{array}{c}\mathrm{kJ} / \mathrm{kg}-\mathrm{K} \\
(\mathrm{Btu} / \mathrm{lb}-\mathrm{F})\end{array}$ & $\begin{array}{c}\mathrm{m}^{2}-\mathrm{K} / \mathrm{W} \\
\left(\mathrm{hr}-\mathrm{ft}^{2}-\mathrm{F} / \mathrm{Btu}\right)\end{array}$ \\
\hline Gypsum board & $\begin{array}{l}0.0127 \\
(0.042)\end{array}$ & $\begin{array}{c}0.160 \\
(0.0926)\end{array}$ & $\begin{array}{l}800 \\
(50)\end{array}$ & $\begin{array}{l}0.837 \\
(0.2)\end{array}$ & $\begin{array}{l}0.079 \\
(0.45)\end{array}$ \\
\hline
\end{tabular}

Window:

\begin{tabular}{|l|c|c|}
\cline { 2 - 3 } \multicolumn{1}{c|}{} & U-value & SHGC \\
\hline Location & $\begin{array}{c}\mathrm{W} / \mathrm{m}^{2}-\mathrm{K} \\
\left(\mathrm{Btu} / \mathrm{hr}^{-}-\mathrm{ft}^{2}-\mathrm{F}\right)\end{array}$ & \\
\hline St. Louis, Bismarck, and Minneapolis & $\begin{array}{c}3.80 \\
(0.67)\end{array}$ & 0.39 \\
\hline Miami and Phoenix & $\begin{array}{c}7.21 \\
(1.27)\end{array}$ & 0.25 \\
\hline
\end{tabular}

The internal gains for the occupied spaces are calculated based on a total sensible heat gain per day per unit to account for a combination of lights, people and equipment per the Space Conditioning calculation procedure of Standard 90.2 (ASHRAE 2001d). These gains equal 21,100 kJ (20,000 Btu) plus the floor area times $170 \mathrm{~kJ} / \mathrm{m}^{2}\left(15 \mathrm{Btu} / \mathrm{ft}^{2}\right)$ and are scheduled as shown in Figure 10. The total latent heat gains are assumed to be 0.2 times the sensible gains.

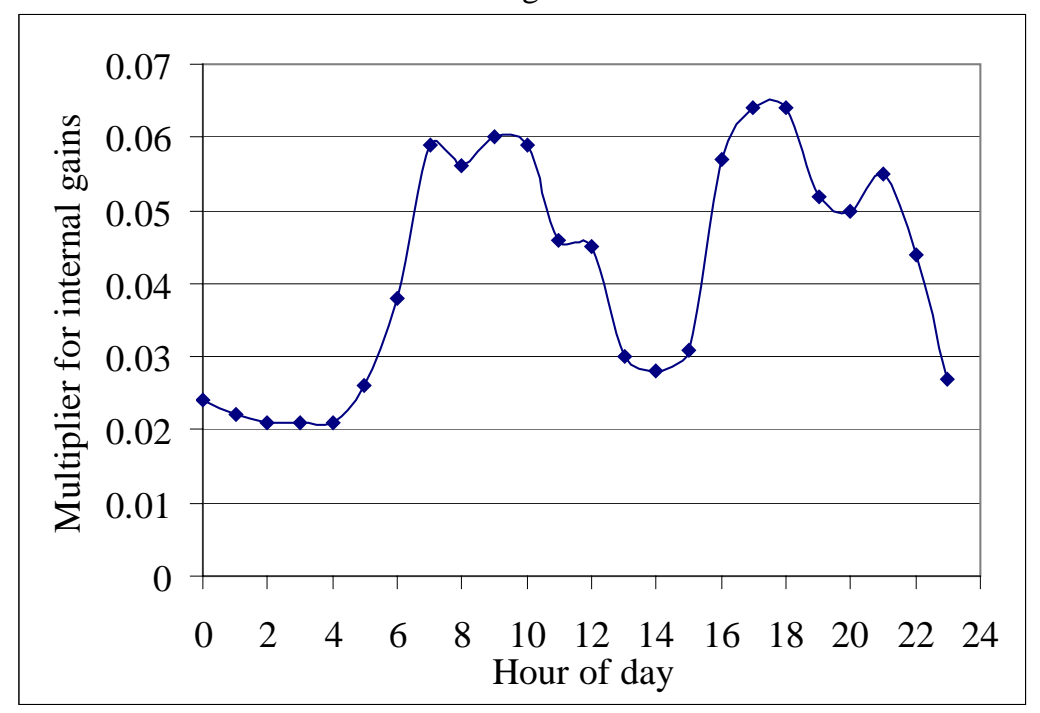

\section{Figure 10 Residential building internal gains schedule}

The thermostats operate on a setpoint with setback/setup basis. The heating setpoint is $20.0^{\circ} \mathrm{C}\left(68{ }^{\circ} \mathrm{F}\right)$ and the cooling setpoint is $25.6^{\circ} \mathrm{C}\left(78{ }^{\circ} \mathrm{F}\right)$. The schedule for the setback/setup for the living units is shown in Figure 11 . The lobby and corridor zones are heated to $20.0^{\circ} \mathrm{C}\left(68^{\circ} \mathrm{F}\right)$ and cooled to $25.6^{\circ} \mathrm{C}\left(78{ }^{\circ} \mathrm{F}\right)$. 


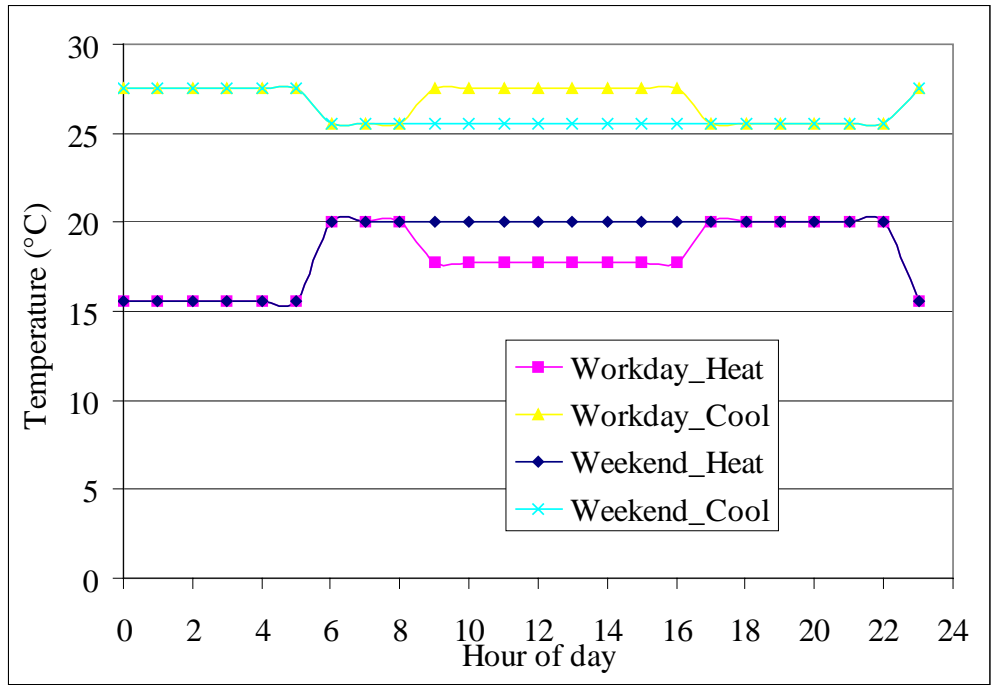

Figure 11 Thermostat schedule for residential building 


\section{Airflow Models}

This section provides the details of the airtightness levels used in the study. Three different airtightness levels (No air barrier, target, and best achievable) were modeled in each building by changing the leakage characteristics in the CONTAM multizone airflow models for each building. The values for the no air barrier level varied for each location, while the target and best achievable construction cases were the same for all locations. The values for the no air barrier (i.e., baseline) case were established through an analysis of the available published airtightness data for buildings other than low-rise residential buildings.

The majority of the data were compiled in a 1998 summary (Persily 1998) supplemented by additional data including more Florida commercial buildings, additional U.K. office buildings, and Canadian apartment buildings added during this study [see references in Appendix A]. The entire dataset of 166 buildings (144 in North America and 22 in U.K.) and the references are presented in Appendix A. This dataset includes all data from the ASHRAE Handbook of Fundamentals (ASHRAE 2001c) but adds data from over 150 additional buildings reported in 13 different studies. Since these data are intended to represent only the baseline case, no buildings known to have been constructed to a specific airtightness level are included. Most of the air leakage rates in the dataset were determined using ASTM E779 fan pressurization tests (ASTM 1999). Others were tested by very similar methods such as Canadian (CGSB 1986 and CGSB 1999), International (ISO 1996) or British (CIBSE 2000) standards. Proskiw and Philips (2001) summarize and compare these and other current or proposed building airtightness testing methods.

As reference points, average airtightness levels from various subsets of the data, in units of $\mathrm{L} / \mathrm{s}-\mathrm{m}^{2}\left(\mathrm{cfm} / \mathrm{ft}^{2}\right)$ at an indoor-outdoor pressure difference of $75 \mathrm{~Pa}\left(0.3\right.$ in $\left.\mathrm{H}_{2} \mathrm{O}\right)$, normalized by above-grade envelope surface area are as follows:

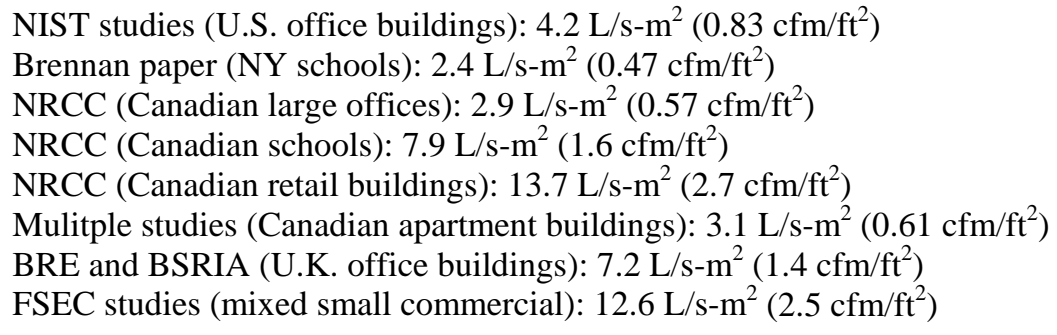

Based on the available information, the dataset was reduced by excluding buildings older than 1960 (even though examination of the data by U.S., Canadian and U.K. authors have found no trends toward increased airtightness in more recent buildings [Persily 1998, Proskiw and Phillips 2001, and Potter et al. 1995]), all industrial buildings, and one extremely leaky building. The data were then divided into north (Standard 90.1 climate zones 5 and above) and south (Standard 90.1 climate zones 4 and below) subsets for North American buildings only. Unfortunately, the available data are inadequate to support a breakdown by the individual climate zones. Finally, within those North and South subsets, average airtightness was calculated for short buildings ( 3 stories and less) and tall buildings (4 stories and up) as the data demonstrate that the tall buildings are tighter on average. The average airtightness values (again in units of $\mathrm{L} / \mathrm{s}-\mathrm{m}^{2}$ at $75 \mathrm{~Pa}$ ( $\mathrm{cfm} / \mathrm{ft}^{2}$ at 0.3 in $\mathrm{H}_{2} \mathrm{O}$ ), normalized by above-grade envelope surface area) are as follows:

North_short (29 buildings): $6.6 \mathrm{~L} / \mathrm{s}-\mathrm{m}^{2}\left(1.3 \mathrm{cfm} / \mathrm{ft}^{2}\right)$

South_short (74 buildings): $11.8 \mathrm{~L} / \mathrm{s}-\mathrm{m}^{2}\left(2.3 \mathrm{cfm} / \mathrm{ft}^{2}\right)$

Based on consideration of the available data, the average measured value from the short buildings in the south was used as the baseline value in the warmest climate (Miami) and the average measured value from the short buildings in the north was used as the baseline value in the coldest climate (Bismarck). The values for the remaining locations were assigned by linearly interpolating between these values using the number of heating degree days (HDD) for the location. As a result, the whole building air leakage values used were (in units of $\mathrm{L} / \mathrm{s}-\mathrm{m}^{2}$ at $75 \mathrm{~Pa}\left(\mathrm{cfm} / \mathrm{ft}^{2} @ 0.3 \mathrm{inH}_{2} \mathrm{O}\right)$ and normalized by above-grade envelope surface area) as follows: 


$\begin{array}{ll}\text { No air barrier: } & \\ \text { Miami } & 11.8 \mathrm{~L} / \mathrm{s}-\mathrm{m}^{2}\left(2.3 \mathrm{cfm} / \mathrm{ft}^{2}\right) \\ \text { Phoenix } & 11.1 \mathrm{~L} / \mathrm{s}-\mathrm{m}^{2}\left(2.2 \mathrm{cfm} / \mathrm{ft}^{2}\right) \\ \text { St. Louis } & 9.1 \mathrm{~L} / \mathrm{s}-\mathrm{m}^{2}\left(1.8 \mathrm{cfm} / \mathrm{ft}^{2}\right) \\ \text { Minneapolis } & 7.2 \mathrm{~L} / \mathrm{s}-\mathrm{m}^{2}\left(1.4 \mathrm{cfm} / \mathrm{ft}^{2}\right) \\ \text { Bismarck } & 6.6 \mathrm{~L} / \mathrm{s}-\mathrm{m}^{2}\left(1.3 \mathrm{cfm} / \mathrm{ft}^{2}\right)\end{array}$

In addition to the baseline level, all buildings were modeled at two levels of increased airtightness. Both published building airtightness data and current commercial buildings airtightness standards were considered in selecting these levels. The 'target' level was selected to represent a level of airtightness that can be achieved through good construction practice, while the 'best achievable' level is based on the tightest levels reported for nonresidential buildings. Achieving the tightest level would require an aggressive program of quality control during construction and airtightness testing, combined with efforts to identify and repair any leaks.

$$
\begin{array}{ll}
\text { Target: } & 1.2 \mathrm{~L} / \mathrm{s}-\mathrm{m}^{2}\left(0.24 \mathrm{cfm} / \mathrm{ft}^{2}\right) \\
\text { Best achievable: } & 0.2 \mathrm{~L} / \mathrm{s}-\mathrm{m}^{2}\left(0.04 \mathrm{cfm} / \mathrm{ft}^{2}\right)
\end{array}
$$

About $6 \%$ of the tested buildings listed in Appendix A would meet the $1.2 \mathrm{~L} / \mathrm{s}-\mathrm{m}^{2}\left(0.24 \mathrm{cfm} / \mathrm{ft}^{2}\right)$ selected target airtightness level. Note that none of these tested buildings was built to an airtightness standard. For comparison, the average reported airtightness level was calculated for non-residential buildings in the U.K., Ireland, and Germany that were constructed to specified whole building airtightness targets as reported in Building Services Journal articles and elsewhere (Anon. 1998, Anon. 2002, Anon. 2003, Cohen 2003, Olivier 2001and Kennett 2004). These 14 buildings, which were of various envelope construction types (curtain wall, masonry, frame, and mixed) and ranged from 1 story to 6 stories, averaged $1.3 \mathrm{~L} / \mathrm{s}-\mathrm{m}^{2}$ at $75 \mathrm{~Pa}\left(0.25 \mathrm{cfm} / \mathrm{ft}^{2}\right.$ at $\left.0.3 \mathrm{inH}_{2} \mathrm{O}\right)$.

In addition to simulations at these levels, the 'no air barrier' and target levels were varied as part of a sensitivity analyses, as described below.

Although the whole building air leakage dataset in Appendix A lacks data for U.S. apartment buildings, a literature review by Edwards (1999) reported air leakage for apartment units in 16 U.S. buildings from four studies (Modera et al. 1985, Diamond et al. 1986, Synertech 1987, Feustel and Diamond 1996). The average leakage of these 16 buildings was $20 \mathrm{~cm}^{2}$ per $\mathrm{m}^{2}\left(0.29 \mathrm{in}^{2}\right.$ per $\left.\mathrm{ft}^{2}\right)$ of floor area at $4 \mathrm{~Pa}$. For the apartment building modeled in this study (and assuming uniform distribution over all apartment surfaces), this value corresponds to $11.6 \mathrm{~L} / \mathrm{s}-\mathrm{m}^{2}$ at $75 \mathrm{~Pa}\left(2.3 \mathrm{cfm} / \mathrm{ft}^{2}\right.$ at 0.3 in $\left.\mathrm{H}_{2} \mathrm{O}\right)$, which is leakier than the average value used in the models discussed above. Also, no direct leakage between adjacent units was assumed for the apartment building. Suite access doors from the hallways were modeled as an effective leakage area of $200 \mathrm{~cm}^{2}\left(31 \mathrm{in}^{2}\right)$ at 4 Pa based on measurements by Wray et al. (1998). 


\section{System Models}

The HVAC systems modeled were specified to be representative of systems that would be installed in typical practice. The systems were different for the different building types and were sized for the appropriate locations. Whether each system included an economizer for the different locations was based on the criteria in ASHRAE 90.1.

\section{Office Building:}

The office building system included water-source heat pumps (WSHPs) with a cooling tower and a boiler serving the common loop. Each zone had its own WSHP rejecting/extracting heat from the common loop. The outdoor air for each zone was supplied to each individual heat pump, and the heat pump blower was on at all times when the zone was occupied. When the location of the building required an economizer, the outdoor air controls were applied to the individual heat pump's airflow. With this approach, different heat pumps could have a different percentage of outdoor air at the same time depending on the loads. For the five modeled locations, St. Louis, Bismarck and Phoenix included economizers and Minneapolis and Miami did not. Return airflow was specified to equal $95 \%$ of supply airflow.

Retail Building:

The retail building system was a packaged rooftop unit including a DX cooling coil and a gas furnace, with a separate system for each individual zone. The required outdoor air was provided by each individual unit so the blower was on at all times when the zone was occupied. When the location of the building required an economizer the outdoor air controls were applied to the individual unit's airflow. In this manner different units could have a different percentage of outdoor air at the same time depending on the loads. For the selected locations, St. Louis, Bismarck and Phoenix included economizers and Minneapolis and Miami did not. Return airflow was specified to equal $95 \%$ of supply airflow.

\section{Residential Building:}

The systems are modeled using manufacturer's data for a residential DX coil with gas furnace, with a separate system for each individual zone. No outdoor air is provided by the system, and therefore no economizer system is modeled. For the gas furnace a simple unit efficiency is used to calculate the required gas input and the heat output for each timestep that requires heating. (This is around $80 \%$ for all the units.) For cooling, the manufacturer's data has corrections for the coil performance and energy consumption based on air conditions at the condenser and evaporator coils. These were single speed units that operate on a on/off basis rather than a reduced speed basis. The simulation uses 5 min timesteps so the minimum time that a unit is on is $5 \mathrm{~min}$. (These units range from 12.0 to 13.25 SEER at ARI 210 rating conditions.) Also, exhaust ventilation will be modeled for each unit with the following values and schedules:

Bathroom exhaust: 23.6 L/s (50 cfm) from 7:00 a.m. to 7:30 a.m. daily

Kitchen exhaust: $47.2 \mathrm{~L} / \mathrm{s}(100 \mathrm{cfm})$ from 6:00 p.m. to 7:00 p.m. daily

Dryer exhaust: $118 \mathrm{~L} / \mathrm{s}(250 \mathrm{cfm})$ from 1:00 p.m. to 4:00 p.m. on Saturday only 


\section{Scalar Calculation}

ASHRAE Standing Standard Project Committee 90.1 has historically selected, and then implemented, energy conservation measures for inclusion in the standard on the basis of a reasonable return on investment in terms of energy saved vs. first cost using life cycle cost economic analysis. In order to apply uniform rules across the different requirements of the standard, whether they are insulation levels or lighting efficiency, a uniform rule is applied to all measures according to whole building energy analysis of prototypical buildings. This rule is based on a multiplier for the energy saved that includes mulitple economic factors, such as the number of years, the cost of money and interest rates. The dimensionless multiplier is termed a Scalar Ratio by the 90.1 committee. At the time of update of the standard from ASHRAE 90.1-1989 to ANSI/ASHRAE/IESNA Standard 90.1- 1999, the development of the scalar ratio and its application across disciplines was documented in McBride (1995) and the value used in this update process was " 8 ".

This life cycle cost methodology was employed in this study to assess the impact of an air barrier requirement as simulated. Specifically, the Scalar Ratio pass-fail methodology used when considering requirements for potential inclusion in ANSI/ASHRAE/IESNA 90.1 was employed, i.e., the value must be less than or equal to a scalar of 8 based on the following equation:

$\left(\mathrm{FYSh}^{*} \mathrm{Ph}+\mathrm{FYSc} * \mathrm{Pc}\right) * 8=>\Delta \mathrm{FC}$

where: $\quad$ FYSh $=$ First year savings in heating (therms)

$\mathrm{Ph}=$ Cost of heating energy $(\$$ per therm)

FYSc $=$ First year savings in cooling $(\mathrm{KWh})$

$\mathrm{Pc}=$ Cost of cooling energy $(\$$ per $\mathrm{KWh})$

$\Delta \mathrm{FC}=$ Cost premium for the energy conservation measure $(\$)$

The actual scalar for each city was then calculated as:

$\Delta \mathrm{FC} /(\mathrm{FYSh} * \mathrm{Ph}+\mathrm{FYSc} * \mathrm{Pc})=\mathrm{Sac}$

where: $\quad$ Sac $=$ Actual scalar

The values used for the cost premium $\Delta \mathrm{FC}$ were estimated as described below, and were employed to allow application of the methodology for the purposes of this study. They are not intended to represent absolute or standardized cost values; the cost values, like the pass-fail scalar ratio, used by SSPC 90.1 are ultimately a committee decision.

Cost of Energy

The development of the Standard 90.1 envelope criteria is predicated on the use of national average prices for the heating and cooling energies. The fundamental issue is that the buildings covered by the standard consist of high-rise residential, commercial and warehouses. Furthermore, each building type can have different energy rate schedules, use different energy sources and have a different type of HVAC equipment or system. The solution to this problem used by the 90.1 committee has been the development of blended heating and cooling energy prices. National averages for electricity and gas were calculated from 2005 Energy Information Agency data for natural gas, electricity and fuel oil for both residential and commercial customers. The national average prices are then weighted by the level of construction activity, energy intensities and end uses, and current efficiencies for the HVAC equipment and forced air distribution systems. The energy price for heating consisted of a blending of $75 \%$ natural gas, $14 \%$ fuel oil and $11 \%$ electricity. The final blended heating price was $\$ 1.01$ per therm. The cooling energies investigated were electricity (98.3 \%) and natural gas $(1.7 \%)$ but a decision was made to use just the electricity cost. The final blended cooling price was $\$ 0.0827$ per kWh of electricity.

\section{Cost Estimate of the Continuous Air Barrier}

There are many possible materials and technologies currently available to construct a building envelope that will meet the whole building target discussed above. The proposal being considered by the 90.1 
committee would allow buildings to meet the air barrier requirement through any of three paths: 1) a material airtightness specification, 2) an assembly airtightness specification, or 3) a whole building airtightness specification. The cost estimates were based on selected options to meet the proposed material airtightness level $\left(0.02 \mathrm{~L} / \mathrm{s} . \mathrm{m}^{2}\right.$ at $75 \mathrm{~Pa}\left[0.004 \mathrm{cfm} / \mathrm{ft}^{2}\right.$ at $\left.\left.0.3 \mathrm{inH}_{2} \mathrm{O}\right]\right)$ that is judged to be consistent with the whole building target used in the energy modeling.

A cost estimate for both the frame building (option 1 below) and the masonry building was obtained using average labor rates from an experienced contractor (TWC 2004) licensed by the Air Barrier Association of America (ABAA). A second estimate was obtained from an independent estimator (HFG 2004). The two estimates were then reconciled to determine the value used in this analysis. A contractor licensed by a national manufacturer of a housewrap product made a third estimate for the frame building (option 2 below, Spinu 2004). Sealing the wall air barrier to the windows, foundation and roof or ceiling air barrier was excluded from the estimate since they are required under 90.1 presently. It was assumed that these existing requirements would be met and the manufacturers' instructions followed.

The three tightening approaches considered for the air barrier, and used in the cost estimates presented in Table 5, are:

1. Masonry Back-up Wall Building:

Standard practice in the industry is to use a damp-proofing material on the concrete block in the cavity behind the brickwork. Asphaltic damp-proofing can dry and crack over time due to shrinkage after evaporation of the volatile solvents. Continuous air barrier requirements for durability can be achieved using a polymer-modified bituminous elastomeric liquid-applied air barrier product, tested to the maximum air leakage requirements of the proposed material $\left(0.02 \mathrm{~L} / \mathrm{s} . \mathrm{m}^{2}\right.$ at $75 \mathrm{~Pa}\left[0.004 \mathrm{cfm} / \mathrm{ft}^{2}\right.$ at $\left.\left.0.3 \mathrm{inH}_{2} \mathrm{O}\right]\right)$. The cost difference between the two materials is included in Table 4.

2. Frame building:

(Option 1). Since typical housewrap does not meet the requirements for maximum air leakage of air barrier materials in the proposed change to the 90.1 standard and gypsum sheathing does (Bombaru et al. 1988), the cost of taping the sheathing joints with a durable tape was estimated for each building.

(Option 2). This option is an upgrade from residential quality housewrap material to a commercial grade wrap that would meet the proposed air barrier material requirements. An upgrade cost (Spinu 2004) of $\$ 0.028 / \mathrm{ft}^{2}$ multiplied by the gross exterior wall area was used, with a $10 \%$ waste and overlap factor added.

The final cost estimates used for analyzing the target cases in the study are summarized in Table 5. As discussed above there are other options that may be used to meet the proposed new criteria for the 90.1 standard.

In order to achieve the target air barrier air leakage rate, normal attention to design, construction and enforcement is expected to become standard practice after a period of education. However, for the best achievable case used in the study, additional expenditure would have to be included for quality assurance and quality control (i.e., for inspections and testing) as shown in Table 4.

Table 4 Estimated Air Barrier Costs (in dollars)

\begin{tabular}{|l|c|c|c|c|}
\hline & $\begin{array}{l}\text { Target Case- } \\
\text { Masonry }\end{array}$ & $\begin{array}{l}\text { Target Case - } \\
\text { Frame (Option 1) }\end{array}$ & $\begin{array}{l}\text { Target Case- } \\
\text { Frame (Option 2) }\end{array}$ & $\begin{array}{l}\text { Additional QA/QC for } \\
\text { Best Achievable Case }\end{array}$ \\
\hline Office & 12054 & 4612 & 325 & 5795 \\
\hline Retail & 7287 & 2604 & 176 & 4745 \\
\hline Apartment & NA & 5317 & 370 & 8153 \\
\hline
\end{tabular}




\section{Results}

The annual gas use, electrical use and average infiltration predicted for the office building is presented in Table 5. The annual average infiltration rate with the baseline air leakage ranges from $0.17 \mathrm{~h}^{-1}$ to $0.26 \mathrm{~h}^{-1}$ depending on the climate. Reducing the air leakage rate to the target level results in annual average infiltration rates ranging from $0.02 \mathrm{~h}^{-1}$ to $0.05 \mathrm{~h}^{-1}$ for an average reduction in infiltration of $83 \%$. Further tightening of the building envelope to the best achievable level essentially eliminates infiltration for the office building. There were no differences in average infiltration between the frame and masonry buildings, and only small differences between the masonry and frame buildings for gas and electricity use for heating and cooling. Table 6 summarizes the annual energy cost savings for the office building at the target air leakage level relative to the baseline level. The annual cost savings are largest in the heating dominated climates with potential gas savings of greater than $40 \%$ and electrical savings of greater than $25 \%$. 
Table 5 Annual gas use, electrical use and annual average infiltration for office building

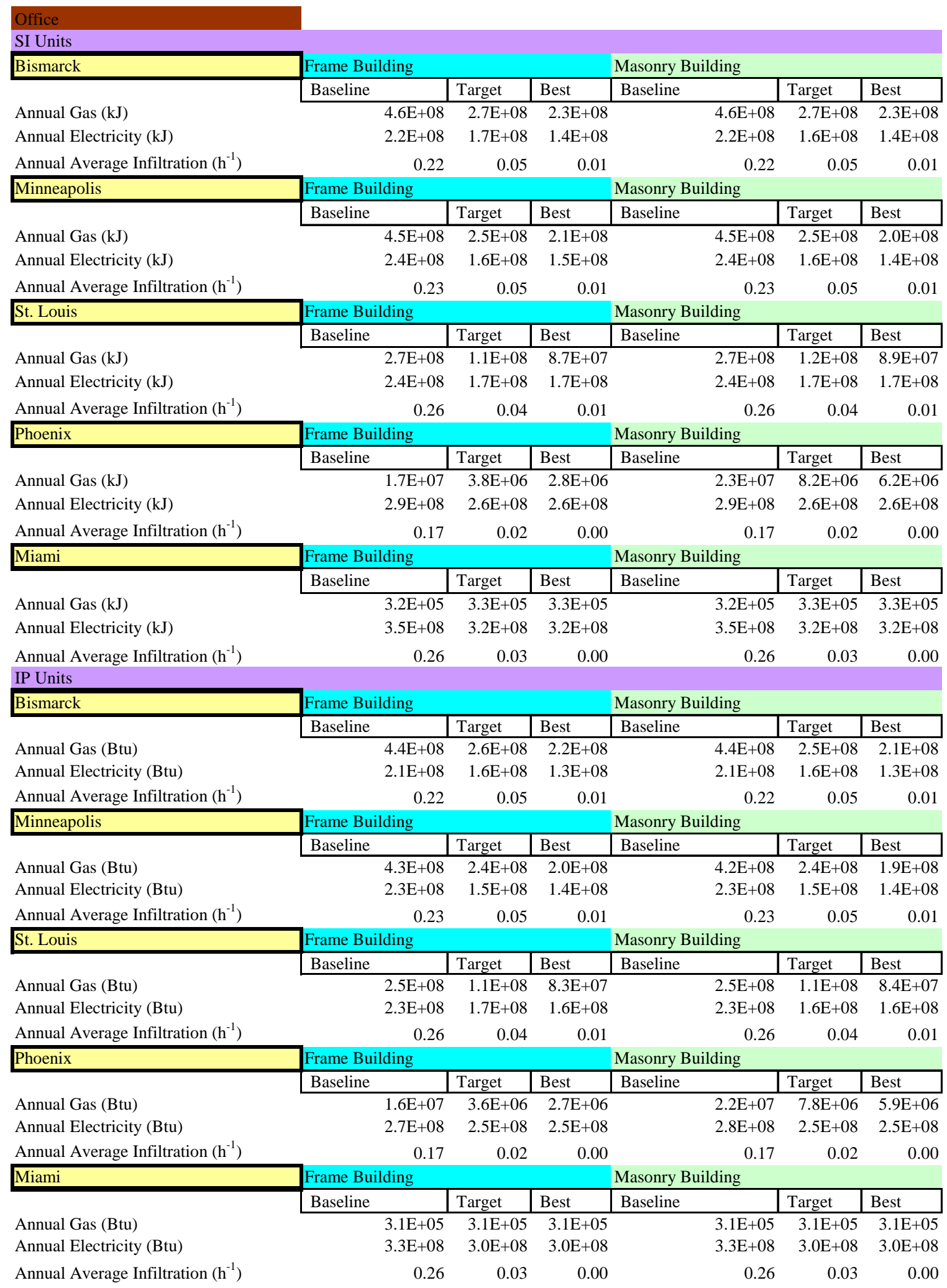


Table 6 Energy cost savings for office building

\begin{tabular}{|l|r|r|r|r|l|}
\hline & \multicolumn{2}{|l|}{$\begin{array}{l}\text { Gas } \\
\text { Savings }\end{array}$} & \multicolumn{2}{l|}{$\begin{array}{l}\text { Electrical } \\
\text { Savings }\end{array}$} & $\begin{array}{l}\text { Total } \\
\text { Savings }\end{array}$ \\
\hline Bismarck & $\$ 1,854$ & $42 \%$ & $\$ 1,340$ & $26 \%$ & $\$ 3,195$ \\
\hline Minneapolis & $\$ 1,872$ & $43 \%$ & $\$ 1,811$ & $33 \%$ & $\$ 3,683$ \\
\hline St. Louis & $\$ 1,460$ & $57 \%$ & $\$ 1,555$ & $28 \%$ & $\$ 3,016$ \\
\hline Phoenix & $\$ 124$ & $77 \%$ & $\$ 620$ & $9 \%$ & $\$ 745$ \\
\hline Miami & $\$ 0$ & $0 \%$ & $\$ 769$ & $10 \%$ & $\$ 769$ \\
\hline
\end{tabular}

\section{Retail building}

Table 7 presents the annual gas use, electrical use and average infiltration predicted for the retail building. The annual average infiltration for the retail building with the baseline air leakage ranges from $0.13 \mathrm{~h}^{-1}$ to $0.24 \mathrm{~h}^{-1}$. Reducing the air leakage rate to the target level almost eliminates infiltration, with all climates having an average rate of less than $0.02 \mathrm{~h}^{-1}$. Further tightening of the building envelope to the best achievable level completely eliminates infiltration for the retail building. Again, there were very few differences between the frame and masonry buildings in either average infiltration or energy. Table 8 summarizes the annual energy cost savings for the retail building at the target air leakage level relative to the baseline level. Unlike the office building, the predicted cost savings for the retail building are fairly independent of climate. The electrical savings in the hot climates are about as large as the gas savings in the cold climates in absolute terms. 
Table 7 Annual gas use, electrical use and annual average infiltration for retail building

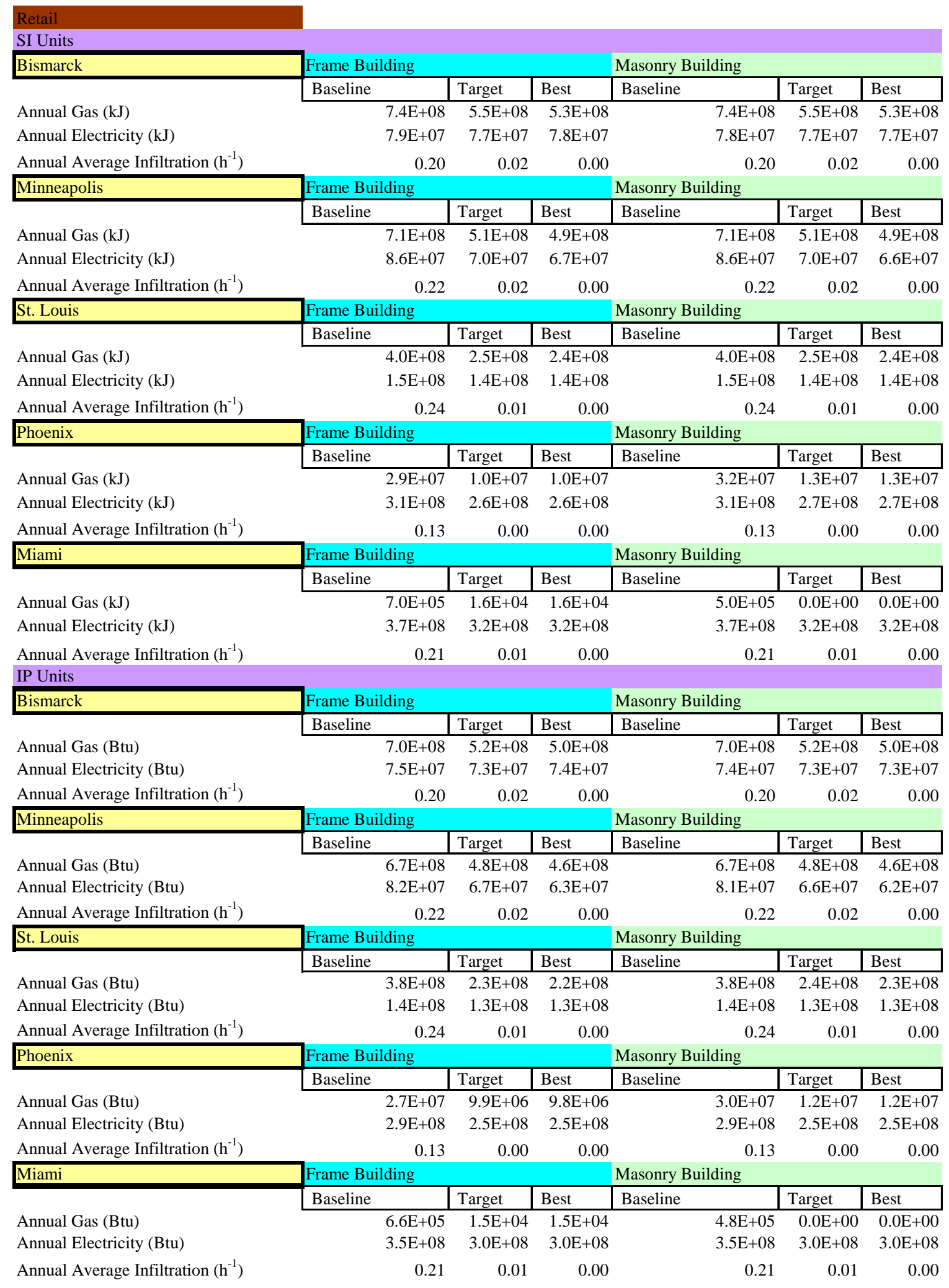


Table 8 Energy cost savings for retail building

\begin{tabular}{|c|c|c|c|c|c|c|}
\hline \multirow{2}{*}{$\begin{array}{l}\text { City } \\
\text { Bismarck }\end{array}$} & \multicolumn{2}{|c|}{ Gas Savings } & \multicolumn{2}{|c|}{$\begin{array}{l}\text { Electrical } \\
\text { Savings }\end{array}$} & \multicolumn{2}{|l|}{\begin{tabular}{|l} 
Total \\
Savings
\end{tabular}} \\
\hline & $\$ 1,835$ & $26 \%$ & $\$ 33$ & $2 \%$ & & $\$ 1,869$ \\
\hline Minneapolis & $\$ 1,908$ & $28 \%$ & $\$ 364$ & $18 \%$ & & $\$ 2,272$ \\
\hline St. Louis & $\$ 1,450$ & $38 \%$ & $\$ 298$ & $9 \%$ & & $\$ 1,748$ \\
\hline Phoenix & $\$ 176$ & $64 \%$ & $\$ 992$ & $14 \%$ & & $\$ 1,169$ \\
\hline Miami & $\$ 6$ & $98 \%$ & $\$ 1,224$ & $14 \%$ & & $\$ 1,231$ \\
\hline
\end{tabular}

\section{Residential building}

Table 9 presents the annual gas use, electrical use and average infiltration predicted for the apartment building. The annual average infiltration rate for the apartment building with the baseline air leakage ranges is slightly higher than the other buildings and ranges from $0.19 \mathrm{~h}^{-1}$ to $0.26 \mathrm{~h}^{-1}$. Reducing the air leakage to the target level results in an average reduction in infiltration of $64 \%$. Further tightening of the building envelope to the best achievable level further reduces the infiltration by an average of $33 \%$. The infiltration remains higher in the tighter apartment buildings relative to the other buildings due to the lack of a mechanical system pressurization effect. The clapboard siding and masonry veneer buildings were quite similar with the masonry building resulting in slightly lower gas use.

Table 10 summarizes the annual energy cost savings for the apartment building at the target air leakage level relative to the baseline level. Similar to the office building, the predicted cost savings for the apartment building are largest in the cold climates with gas savings of $40 \%$ or more. 
Table 9 Annual gas use, electrical use and annual average infiltration for apartment building

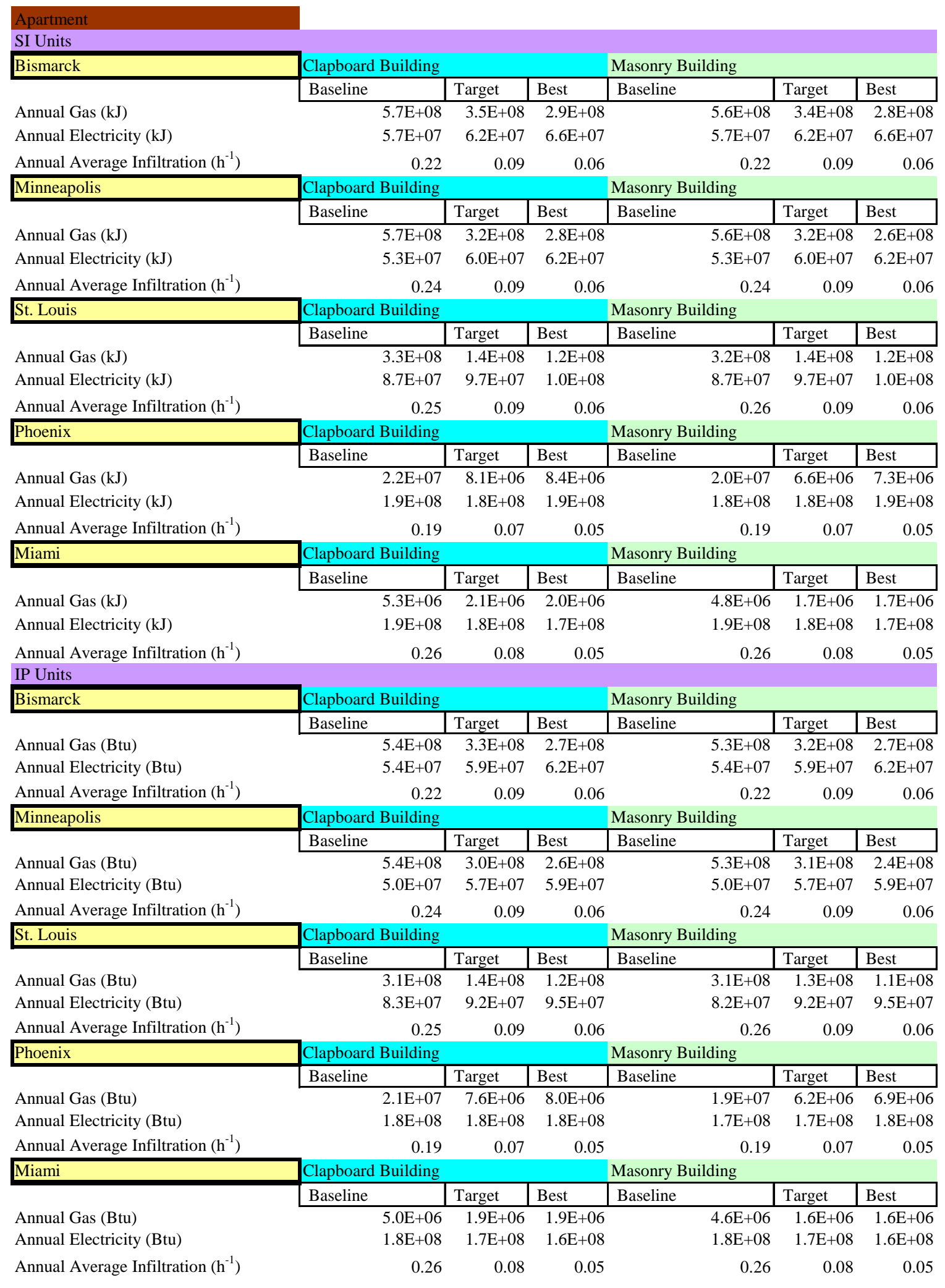


Table 10 Energy cost savings for apartment building

\begin{tabular}{|l|r|r|r|r|r|}
\hline & \multicolumn{1}{l|}{$\begin{array}{l}\text { Gas } \\
\text { Savings }\end{array}$} & \multicolumn{2}{l|}{$\begin{array}{l}\text { Electrical } \\
\text { Savings }\end{array}$} & $\begin{array}{l}\text { Total } \\
\text { Savings }\end{array}$ \\
\hline Bismarck & $\$ 2,187$ & $40 \%$ & $-\$ 116$ & $-9 \%$ & \\
\hline Minneapolis & $\$ 2,421$ & $43 \%$ & $-\$ 165$ & $-14 \%$ & $\$ 2,071$ \\
\hline St. Louis & $\$ 1,794$ & $57 \%$ & $-\$ 232$ & $-12 \%$ & $\$ 2,256$ \\
\hline Phoenix & $\$ 133$ & $65 \%$ & $\$ 0$ & $0 \%$ & $\$ 1,562$ \\
\hline Miami & $\$ 31$ & $63 \%$ & $\$ 380$ & $9 \%$ & $\$ 133$ \\
\hline
\end{tabular}

Unlike the retail and office buildings, tightening the residential building envelope in the cooler climates resulted in a predicted electrical cost penalty of up to $14 \%$. In all of the building types, there are some hours where the reduction of infiltration eliminates a 'free cooling' effect during which time the cool outdoor air offsets the internal heat gain of the building. However, in the apartment building for these cooler climates, this impact summed over the course of the year more than offsets the impact of lower infiltration during hot hours when it adds to the cooling load. There are key differences between the building types that produce this effect. First, the apartment building lacks both an economizer to purposefully take advantage of free cooling effect. In fact, the apartment also lacks continuous ventilation to coincidentally take advantage of the free cooling effect. Second, the apartment building cooling setpoints are higher during the day and lower at night, which results in smaller cooling loads during the hottest hours and larger cooling hours during the cooler hours. However, it is likely that some of the predicted electrical use for cooling in the residential building would not occur in the real world because part of the free cooling effect happens during winter or shoulder seasons when residents may not operate their air-conditioning and would open windows for the free cooling. Figure 12 demonstrates this effect for the apartment building in Phoenix. The tighter target air leakage level results in predicted increases in cooling energy use from October through April but reduces cooling energy in the hottest months of June through September. The average savings during these hot months is $10 \%$. 


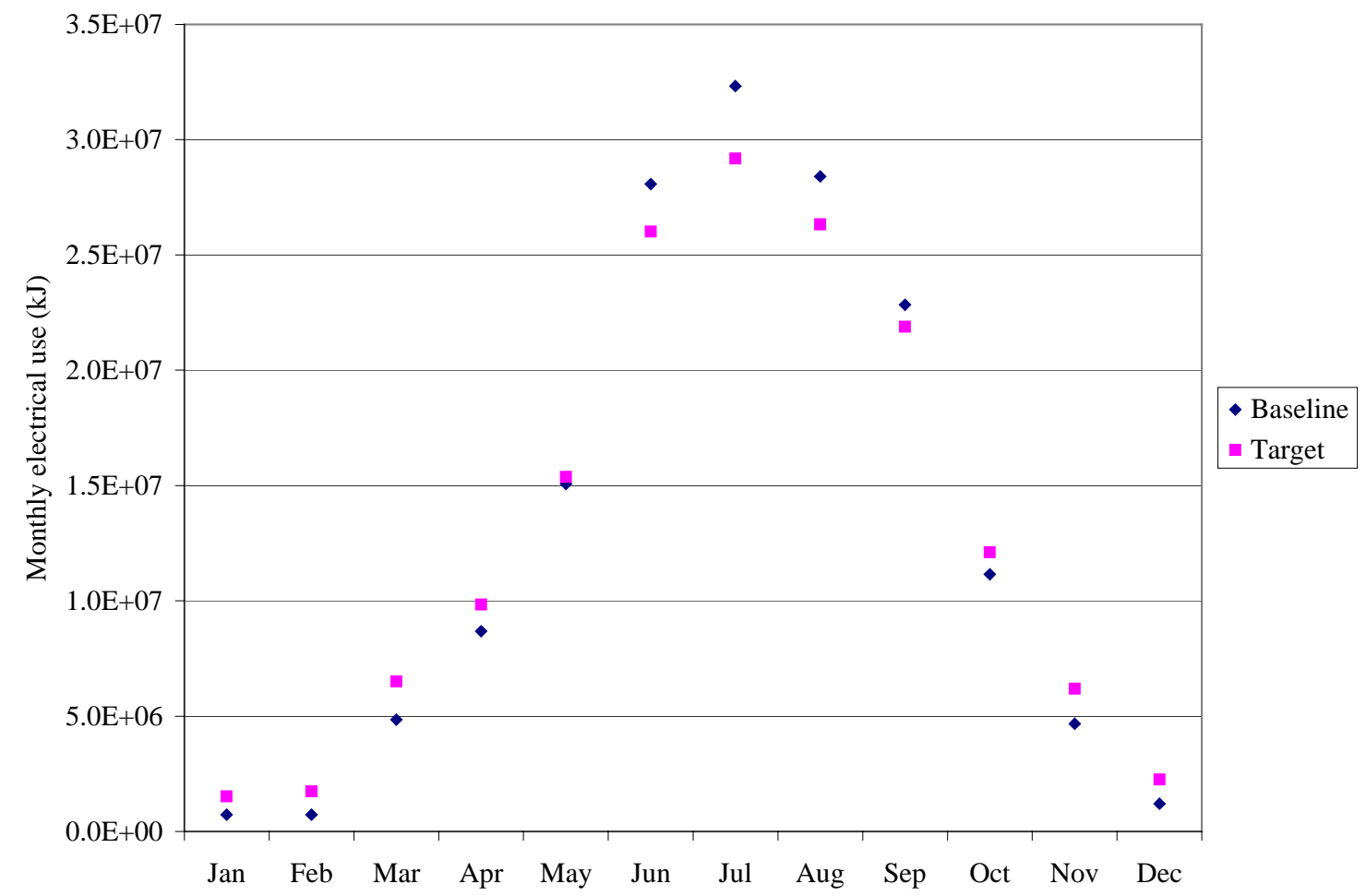

Figure 12 Monthly electrical use in Phoenix apartment building

\section{Comparison to CBECS-derived benchmarks}

Since these prototype buildings are not based on an actual building, it is not possible to directly validate the model predictions. However, it is valuable to compare the predictions to available benchmarks of heating and cooling energy use in comparable U.S. buildings. One option is to compare the predicted annual gas and electricity use to recent Commercial Building Energy Consumption Survey (CBECS) results available from the U.S. Department of Energy. The most recent available commercial energy data available are for 1999 (http://www.eia.doe.gov/emeu/cbecs/detailed_tables_1999.html). The CBECS does not directly measure the portion of energy used in buildings for heating and cooling, but instead uses statistical methods to derive estimates of energy end-use consumption.

For the 1999 CBECS, a new nonlinear regression method was used to estimate the total energy used, with a breakdown by principal building activity. To compare the results from this study, the results for the three baseline buildings were normalized by floorspace area. Similarly, the aggregate energy end-use data for the Office and Retail (Other than Mall) categories were normalized by the closest available floorspace numbers from the 1999 CBECS Building Characteristics tables. The 1999 CBECS value for natural gas used for space heating was normalized by total floorspace area in buildings with gas heating, the value for electricity used for cooling was normalized by total floorspace with cooling, and the value for ventilation was normalized by the total floorspace. CBECS also includes a small amount of electricity used for space heating but this amount was neglected. The normalized values of electricity used for cooling and electricity were then added to obtain the total electricity used for space conditioning.

The values derived from the 1999 CBECS data are presented in Table 11. Note that these values vary considerably from the Commercial Buildings Energy End-Use Intensities listed in the 2004 Buildings Energy Databook (DOE 2004). One cause of the difference is that the Buildings Energy Databook uses older CBECS data from 1995. More significantly, the 1995 CBECS data is based on the discontinued methodology. As explained on the DOE Energy Information Agency website 
(http://www.eia.doe.gov/emeu/cbecs/enduse_consumption/compare.html), applying the new methodology to the 1995 data results in significant changes, presumably improvements, to the energy end-use estimates.

Similarly, normalized national averages for space heating gas and air-conditioning electricity usage in the apartment buildings were calculated from the 2001 U.S. Department of Energy Residential Energy Consumption Survey (RECS) data (http://www.eia.doe.gov/emeu/recs/contents.html). The housing category used was Apartments in Buildings with 5 or More Units and energy usage was based on Units with Natural Gas as the Main Space Heating Fuel for gas and on Units with Central Air-Conditioning for electricity.

Since the CBECS and RECS estimates represent national averages, they are compared to the average of the predicted results for the five locations. While the averaged results from this study should not be interpreted as representing a true national average, the goal of this comparison is not a comprehensive validation but rather to establish the reasonableness of the baseline cases. As seen in Table 11, the average predictions for the baseline cases are all at least $20 \%$ below the values calculated from the 1999 CBECS-derived and 2001 RECS-derived national average data except for the gas used by the retail prototype, which is less than $10 \%$ higher. Considering that the CBECS estimates include buildings of all ages, sizes, construction types, etc., there are many factors that could explain such differences. However since model input decisions were made with the intent of making a conservative prediction, it is not surprising that the predicted values are mostly lower than the CBECS derived values. This indicates that the calculated savings in absolute terms would be even larger than presented above thus resulting in lower scalar values than estimated. While the apartment baseline results are significantly different than the RECS average, the simulation results are similarly lower thus ensuring that the calculated energy savings are not due to reductions in unrealistically large baseline loads.

Table 11 Comparisons of Predicted Gas and Electricity Used to CBECS and RECS Estimates

\begin{tabular}{|c|c|c|c|c|c|c|}
\hline \multirow[b]{2}{*}{ Location } & \multicolumn{2}{|c|}{$\begin{array}{l}\text { Baseline Office } \\
\text { Prototype }\end{array}$} & \multicolumn{2}{|c|}{ Baseline Retail Prototype } & \multicolumn{2}{|c|}{$\begin{array}{l}\text { Baseline Apartment } \\
\text { Prototype }\end{array}$} \\
\hline & $\begin{array}{l}\text { Gas } \\
\text { MJ/m } \mathbf{m}^{2} \\
\left(\mathbf{k B t u} / \mathrm{ft}^{2}\right)\end{array}$ & $\begin{array}{c}\text { Electricity } \\
\mathbf{k W} \cdot \mathbf{h} / \mathbf{m}^{2} \\
\left(\mathbf{k B t u} / \mathbf{f t}^{2}\right)\end{array}$ & $\begin{array}{l}\text { Gas } \\
\mathbf{M J} / \mathbf{m}^{2} \\
\left(\mathbf{k B t u} / \mathbf{f t}^{2}\right)\end{array}$ & $\begin{array}{l}\text { Electricity } \\
\mathbf{k W} \cdot \mathbf{h} / \mathbf{m}^{2} \\
\left(\mathbf{k B t u} / \mathbf{f t}^{2}\right)\end{array}$ & $\begin{array}{l}\text { Gas } \\
\mathbf{M J} / \mathbf{m}^{2} \\
\left(\mathbf{k B t u} / \mathbf{f t}^{2}\right)\end{array}$ & $\begin{array}{l}\text { Electricity } \\
\mathbf{k W} \cdot \mathbf{h} / \mathbf{m}^{2} \\
\left(\mathbf{k B t u} / \mathbf{f t}^{2}\right)\end{array}$ \\
\hline Bismarck & $200(18)$ & $28(9.0)$ & 660 (58) & $19(6.2)$ & 171 (15) & $4.7(1.5)$ \\
\hline Minneapolis & 200 (18) & $30(9.4)$ & 630 (55) & 21 (6.7) & 171 (15) & $4.4(1.4)$ \\
\hline St. Louis & $110(10)$ & 30 (9.6) & $350(31)$ & 38 (12) & $98(8.6)$ & $6.9(2.2)$ \\
\hline Phoenix & $8.0(0.7)$ & 35 (11) & $26(2.3)$ & 76 (24) & $6.8(0.6)$ & $15(4.8)$ \\
\hline Miami & 0 & 44 (14) & $1.1(0.1)$ & 91 (29) & $1.1(0.1)$ & $15(4.9)$ \\
\hline Average & $110(9.4)$ & $35(11)$ & $330(29)$ & $50(16)$ & $88(7.7)$ & $9.4(3.0)$ \\
\hline $\begin{array}{l}1999 \\
\text { CBECS }\end{array}$ & 388 (34) & $82(26)$ & $310(27)$ & $63(20)$ & NA & NA \\
\hline 2001 RECS & NA & NA & NA & NA & $285(25)$ & $22(6.9)$ \\
\hline
\end{tabular}

In addition to the CBECS values, the office building simulation results were also compared to predictions from a Pacific Northwest Laboratories study (Crawley and Schliesing 1992). In this study the DOE-2 building energy simulation tool was used to predict the annual energy consumption for a two-story office building in Greensboro, NC, Tucson, AZ, and Scranton, PA. They found heating gas use of $207 \mathrm{MJ} / \mathrm{m}^{2}$ $\left(18.2 \mathrm{kBtu} / \mathrm{ft}^{2}\right)$ and cooling and fan electricity use of $67.1 \mathrm{~kW} \cdot \mathrm{h} / \mathrm{m}^{2}\left(21.3 \mathrm{kBtu} / \mathrm{ft}^{2}\right)$ for Greensboro, heating energy use of $52.2 \mathrm{MJ} / \mathrm{m}^{2}\left(4.6 \mathrm{kBtu} / \mathrm{ft}^{2}\right)$ and cooling and fan electricity use of $99.2 \mathrm{~kW} \cdot \mathrm{h} / \mathrm{m}^{2}\left(31.5 \mathrm{kBtu} / \mathrm{ft}^{2}\right)$ for Tucson, and heating gas use of $260 \mathrm{MJ} / \mathrm{m}^{2}\left(22.9 \mathrm{kBtu} / \mathrm{ft}^{2}\right)$ and cooling and fan electricity use of $35.3 \mathrm{~kW} \cdot \mathrm{h} / \mathrm{m}^{2}\left(11.2 \mathrm{kBtu} / \mathrm{ft}^{2}\right)$ for Scranton. While there are many differences between the PNL buildings and the assumptions in this study, the gas and electricity use for the baseline office buildings in Table 12 for the closest comparable climates are all conservative compared to the PNL study. 


\section{Cost Effectiveness}

As described earlier, a cost effectiveness analysis of the air barrier energy savings was conducted using the scalar ratio methodology employed by SSPC 90.1. This cost analysis was performed to put the calculated energy savings in context using estimated values of the costs associated with the air barrier measures. As seen in Table 12, the majority of cases with one exception (the office building with masonry backup in climate zones 1 and 2) have a Scalar Ratio less than 8 for the Target case. Based on this criterion, the residential building can use either of the airtightening options outlined in climate zones 3 and higher, but Option 2 is more cost effective in climate zones 1 and 2.

Office building: The masonry building expenditure on the continuous air barrier is cost-effective in climate zoned 3 and higher. The energy savings in climate zones 1 and 2, although significant, is not enough to offset the expenditure for the air barrier within the accepted guidelines of 90.1; in other words, with a Scalar of 16.2 or higher, it does not meet the maximum Scalar Ratio limit of 8. This would imply that an exception for masonry buildings in climate zones 1 and 2 is suggested by the study. The frame building air barrier is cost effective with both airtightening strategies in all climates.

Retail building: The Scalar Ratio calculated for all the climate zones for both the masonry and frame building types indicate that all air barrier strategies are cost-effective.

Multi-Unit Apartment Building: Based on the Scalar Ratio, the air barrier strategy option 1 is not costeffective in climate zones 1 and 2, but the air barrier strategy option 2 is cost-effective in all climates. There is no significant difference between the building with clapboard siding and masonry veneer. 
Table 12 Summary of Calculated Scalar Ratios

\begin{tabular}{|c|c|c|c|c|c|}
\hline Two Story Office Building & Bismarck & Minneapoli & St. Louis & Phoenix & Miami \\
\hline Cost of energy saved x Scalar of 8 & $\$ 25,701$ & $\$ 25,701$ & $\$ 24,122$ & $\$ 5,956$ & $\$ 6,153$ \\
\hline \multicolumn{6}{|l|}{ Masonry Backup Wall } \\
\hline First cost of the air barrier & $\$ 12,054$ & $\$ 12,054$ & $\$ 12,054$ & $\$ 12,054$ & $\$ 12,054$ \\
\hline Calculated Scalar & 3.8 & 3.8 & 4.0 & 16.2 & 15.7 \\
\hline \multicolumn{6}{|c|}{ Steel Frame Building - Taped sheathing (Option 1) } \\
\hline First cost of the air barrier & $\$ 4,612$ & $\$ 4,612$ & $\$ 4,612$ & $\$ 4,612$ & $\$ 4,612$ \\
\hline Calculated Scalar & 1.4 & 1.4 & 1.5 & 6.2 & 6.0 \\
\hline \multicolumn{6}{|c|}{ Steel Frame Building - Commercial Wrap (Option 2) } \\
\hline First cost of the air barrier & $\$ 325$ & $\$ 325$ & $\$ 325$ & $\$ 325$ & $\$ 325$ \\
\hline Calculated Scalar & 0.1 & 0.1 & 0.1 & 0.4 & 0.4 \\
\hline
\end{tabular}

\begin{tabular}{|l|r|r|r|r|r|}
\hline One Story Retail Building & Bismarck & Minneapoli & St. Louis & Phoenix & Miami \\
\hline Cost of energy saved x Scalar of 8 & $\$ 14,946$ & $\$ 18,174$ & $\$ 13,985$ & $\$ 9,345$ & $\$ 9,840$ \\
\hline Masonry Backup Wall & & & & & \\
\hline First cost of the air barrier & $\$ 7,287$ & $\$ 7,287$ & $\$ 7,287$ & $\$ 7,287$ & $\$ 7,287$ \\
\hline Calculated Scalar & 3.9 & 3.2 & 4.2 & $\mathbf{6 . 2}$ & 5.9 \\
\hline Steel Frame Building - Taped sheathing (Option 1) & & & & \\
\hline First cost of the air barrier & $\$ 2,604$ & $\$ 2,604$ & $\$ 2,604$ & $\$ 2,604$ & $\$ 2,604$ \\
\hline Calculated Scalar & $\mathbf{1 . 4}$ & $\mathbf{1 . 1}$ & $\mathbf{1 . 5}$ & $\mathbf{2 . 2}$ & $\mathbf{2 . 1}$ \\
\hline Steel Frame Building - Commercial Wrap (Option 2) & & & & \\
\hline First cost of the air barrier & $\$ 176$ & $\$ 176$ & $\$ 176$ & $\$ 176$ & $\$ 176$ \\
\hline Calculated Scalar & $\mathbf{0 . 1}$ & $\mathbf{0 . 1}$ & $\mathbf{0 . 1}$ & $\mathbf{0 . 2}$ & $\mathbf{0 . 1}$ \\
\hline
\end{tabular}

\begin{tabular}{|c|c|c|c|c|c|}
\hline Four Story Wood Frame Apartment Building (Clapboard Siding) & Bismarck & Minneapolis & St. Louis & Phoenix & Miami \\
\hline Cost of energy saved x Scalar of 8 & $\$ 16,567$ & $\$ 18,045$ & $\$ 12,498$ & $\$ 1,067$ & $\$ 3,294$ \\
\hline \multicolumn{6}{|l|}{ Taped sheathing (Option 1) } \\
\hline First cost of the air barrier & $\$ 5,317$ & $\$ 5,317$ & $\$ 5,317$ & $\$ 5,317$ & $\$ 5,317$ \\
\hline Calculated Scalar & 2.6 & 2.4 & 3.4 & 39.9 & 12.9 \\
\hline \multicolumn{6}{|l|}{ Commercial Wrap (Option 2) } \\
\hline First cost of the air barrier & $\$ 370$ & $\$ 370$ & $\$ 370$ & $\$ 370$ & $\$ 370$ \\
\hline Calculated Scalar & 0.2 & 0.2 & 0.2 & 2.8 & 0.9 \\
\hline Four Story Wood Frame Apartment Building (Masonry Veneer) & Bismarck & Minneapoli & St. Louis & Phoenix & Miami \\
\hline Cost of energy saved x Scalar of 8 & $\$ 16,468$ & $\$ 17,067$ & $\$ 12,326$ & $\$ 994$ & $\$ 3,286$ \\
\hline \multicolumn{6}{|l|}{ Taped sheathing (Option 1) } \\
\hline First cost of the air barrier & $\$ 5,317$ & $\$ 5,317$ & $\$ 5,317$ & $\$ 5,317$ & $\$ 5,317$ \\
\hline Calculated Scalar & 2.6 & 2.5 & 3.5 & 42.8 & 12.9 \\
\hline \multicolumn{6}{|l|}{ Commercial Wrap (Option 2) } \\
\hline First cost of the air barrier & $\$ 370$ & $\$ 370$ & $\$ 370$ & $\$ 370$ & $\$ 370$ \\
\hline Calculated Scalar & 0.2 & 0.2 & 0.2 & 3.0 & \\
\hline
\end{tabular}

Color key:

$$
\begin{array}{r|l}
3.8 & \text { Scalar }=<8 \\
16.2 & \text { Scalar }>8
\end{array}
$$




\section{Sensitivity Analysis}

Although the energy and airflow models used for this study require many input values, two of the most important values in determining the potential cost effectiveness of the proposed air barrier requirements are the baseline and target whole building air leakage values. As seen in Appendix A, the existing air leakage measurement cover a large range of air leakage values. To investigate the impact of the particular values used in the study, sensitivity analyses were performed on each of these values for the frame office building in St. Louis (the middle climate studied). First, the whole building air leakage rate used for the baseline case was varied in steps over the range of $2.8 \mathrm{~L} / \mathrm{s}-\mathrm{m}^{2}$ at $75 \mathrm{~Pa}$ to $9.0 \mathrm{~L} / \mathrm{s}-\mathrm{m}^{2}$ at $75 \mathrm{~Pa}\left(0.55 \mathrm{cfm} / \mathrm{ft}^{2}\right.$ at 0.3 in $\mathrm{H}_{2} \mathrm{O}$ to $1.8 \mathrm{cfm} / \mathrm{ft}^{2}$ at 0.3 in $\mathrm{H}_{2} \mathrm{O}$ ). Scalar ratios were calculated using the predicted energy savings for each baseline level relative to the same target as above (i.e., $1.2 \mathrm{~L} / \mathrm{s}-\mathrm{m}^{2}$ at $75 \mathrm{~Pa}\left(0.24 \mathrm{cfm} / \mathrm{ft}^{2}\right.$ at 0.3 in $\left.\mathrm{H}_{2} \mathrm{O}\right)$ ) and the costs for option 1 in Table 5. As expected, Figure 13 shows the scalar ratio dropping as the baseline leakage increases. In other words, the energy savings of a building at the target airtightness relative to a tighter baseline building is smaller than the energy savings at the target airtightness relative to a leakier baseline building. However, the figure shows that even if the baseline leakage were set far lower than the $9.1 \mathrm{~L} / \mathrm{s}-\mathrm{m}^{2}\left(1.8 \mathrm{cfm} / \mathrm{ft}^{2}\right)$ assumed in this study for St. Louis, the resulting scalar ratio would still be below 8 for this building and climate combination. For example, if the baseline building leakage rate were set at 4.2 $\mathrm{L} / \mathrm{s}-\mathrm{m}^{2}\left(0.8 \mathrm{cfm} / \mathrm{ft}^{2}\right)$, which is the average of U.S. office buildings measured in past NIST studies, then the calculated scalar ratio would be about 3.3.

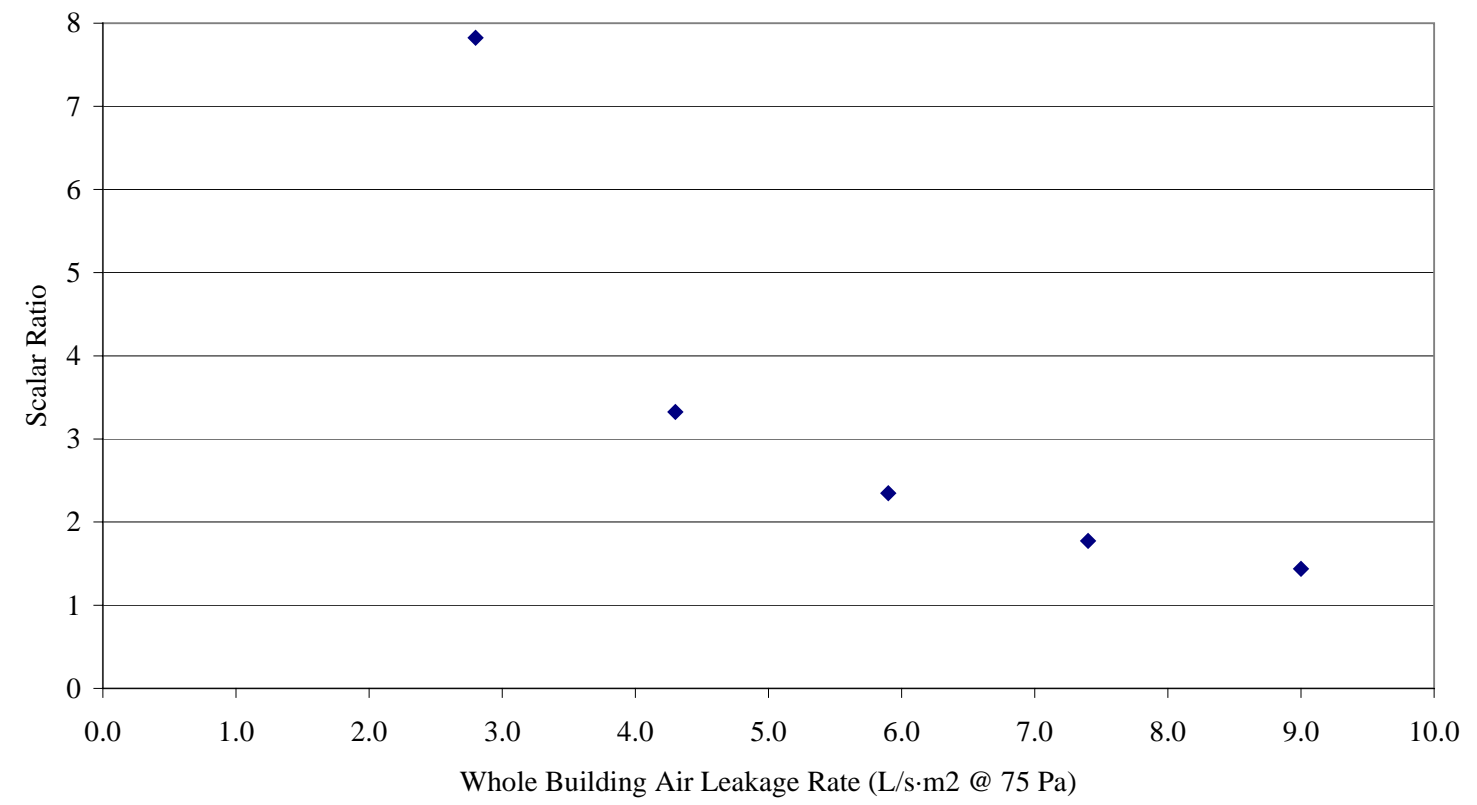

Figure 13 Dependence of scalar ratio on baseline air leakage rate

A similar exercise was performed in which the baseline air leakage rate was held steady at the value used in this study but the target air leakage value was varied over a range of $1.2 \mathrm{~L} / \mathrm{s}-\mathrm{m}^{2}$ at $75 \mathrm{~Pa}$ to $2.8 \mathrm{~L} / \mathrm{s}-\mathrm{m}^{2}$ at 75 $\mathrm{Pa}\left(0.24 \mathrm{cfm} / \mathrm{ft}^{2}\right.$ at 0.3 in $\mathrm{H}_{2} \mathrm{O}$ to $0.55 \mathrm{cfm} / \mathrm{ft}^{2}$ at 0.3 in $\left.\mathrm{H}_{2} \mathrm{O}\right)$. As above, simulations were performed for the frame office building in St. Louis to estimate energy savings and the cost for option 1 in Table 5 were used to calculate the scalar ratios. While the predicted scalar ratio does increase with the target air leakage rate, Figure 14 shows that the scalar ratio is relatively insensitive to the target air leakage rate over the range studied for this building and climate combination. Specifically, if the building with the air barrier is assumed to be 2.33 times as leaky as the target of $1.2 \mathrm{~L} / \mathrm{s}-\mathrm{m}^{2}$ at $75 \mathrm{~Pa}\left(0.24 \mathrm{cfm} / \mathrm{ft}^{2}\right.$ at 0.3 in $\left.\mathrm{H}_{2} \mathrm{O}\right)$ assumed in this study, the predicted scalar ratio increases by only $20 \%$ and remains far below 8 . 


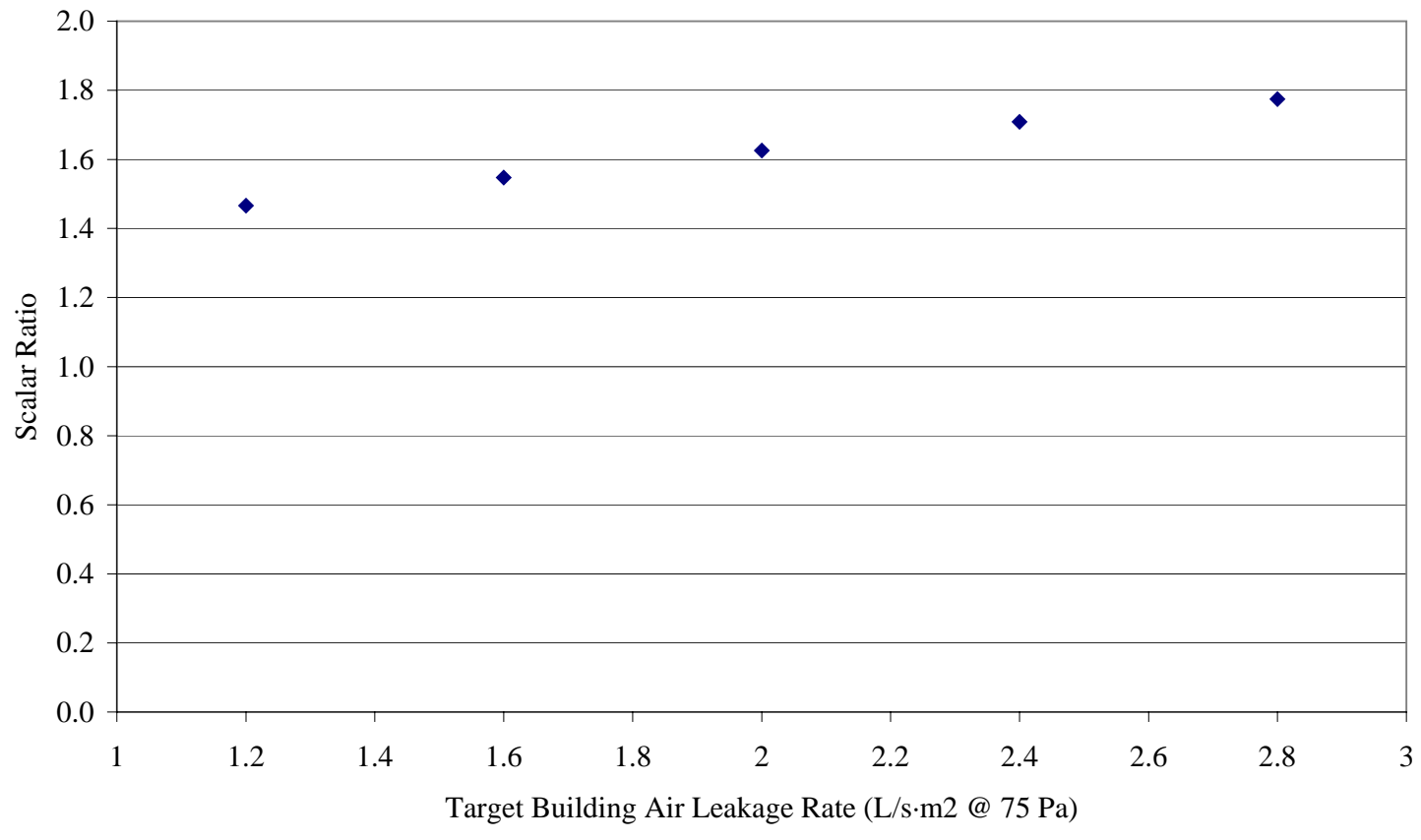

Figure 14 Dependence of scalar ratio on target air leakage rate 


\section{Discussion}

This simulation study included a small number of building types with a specific set of energy-related parameters (i.e., envelope construction types, internal loads, ventilation rates, etc.) in a limited set of climates. Predicted potential annual heating and energy cost savings for these buildings ranged from $2 \%$ to $36 \%$ with the largest savings occurring in the heating-dominated climates of Minneapolis and Bismarck and the smallest savings occurring in the cooling-dominated climates of Phoenix and Miami. The cost effectiveness analysis utilized costs for certain specific materials but other materials may be used to achieve the whole building airtightness target level used in the study.

Only a few other reports of the energy impacts of infiltration in commercial buildings and the potential savings due to tightening could be found for comparison. For the most part, these other studies are not as detailed as the current effort and may employ building configuration, airtightness, and other parameters that vary significantly from this study but they do provide some reference for comparison.

Potter et al. (1995) estimated the heating load due to infiltration for two U.K. office buildings of approximately equal size. They found that a $63 \%$ reduction in air leakage could result in a reduction in annual heating energy loss due to infiltration of about $300 \mathrm{MW} / \mathrm{m}^{2}$. Since whole building energy analysis was not performed, this is not directly equivalent to the heating energy savings predicted in this study but it does concur with the potential large impact of building airtightness on heating energy use.

Edwards (1999) reported a modeling study of the ventilation and infiltration energy impacts in a 10-story apartment building in a range of Canadian climates. This study employed the CONTAM multizone airflow model to create a model of an actual tested building and estimated that infiltration would be responsible for $31 \%$ to $46 \%$ of the average peak heating load (based on measurements in four Toronto apartment buildings reported by Scanada 1991). While the potential savings due to envelope tightening of $40 \%$ to $43 \%$ calculated for Bismarck and Minneapolis predicted in the current study are not directly comparable, the impact of infiltration on heating loads are of a similar magnitude. The Scanada report estimated that infiltration contributed an average of $32 \%$ of the annual heating load in those buildings.

Building Sciences LTD presents an estimate of potential annual heating savings for an industrial building in London of $60 \mathrm{MJ} / \mathrm{m}^{2}$ due to a reduction in envelope leakiness of $75 \%$ (http://www.airleakage.co.uk/why.htm). The gas savings for the target level relative to the baseline level in St. Louis (the closest climate) estimated in this study ranged from $56 \mathrm{MJ} / \mathrm{m}^{2}$ to $130 \mathrm{MJ} / \mathrm{m}^{2}$ depending on the building type. Again, these estimates are for different buildings with different assumptions but the magnitude of savings falls within the same overall range.

Parekh (1992) described measured airtightness and monitored energy before and after sealing efforts in two existing high-rise residential buildings in Canada. The air leakage of the buildings was reduced by an average of $35 \%$, which resulted in an average heating energy consumption reduction of $9 \%$. While the energy saving is smaller than predicted in this study, the difference is readily explained by the modest reduction in air leakage achieved for this retrofit study.

Similarly, Shaw and Reardon (1995) reported an $11 \%$ reduction in monitored heating energy consumption after a $43 \%$ improvement in measured airtightness of a 20-story office building in Ottawa. Again, the measured energy savings is smaller than the estimates in this study but the differences are easily explained by the different building type and the more modest reduction in air leakage achieved in this retrofit study.

As mentioned above, this study included a limited set of building-climate combinations and this work could be extended in a variety of ways. Specific recommendations for future work include:

- Continue study to develop more refined (i.e., climate-specific) airtightness targets,

- Extend study to other building categories,

- Perform factorial analysis to examine the potential interaction between airtightness and other building parameters, 
- Test airtightness of buildings built to a tightness standard (possibly in MA) to evaluate whether tightness targets are being met in practice,

- Analyze the costs and potential energy savings from tightening of existing buildings and develop recommendations for the existing building stock,

- And, develop diagnostic protocols and tools for failures of building envelopes that deteriorate IAQ and energy efficiency.

\section{Acknowledgements}

This work was sponsored by the US. Department of Energy, Office of Building Technologies under Interagency Agreement No. DE-AI01-01EE27615. The authors wish to acknowledge the efforts of Terry Logee of DOE, Merle McBride and the ASHRAE SSPC90.1 Envelope Subcommittee in support of this project. 


\section{References}

Anis, W. 2001. “The Impact of Airtightness on System Design”, ASHRAE Journal Vol. 43, No. 12.

Anon. 2002. "Monitored performance of an innovative multi-unit residential building" Canada Mortgage and Housing Corporation Research Highlight.

Anon. 1998. “PROBE 14: Elizabeth Fry Building”, Building Services Journal, April 1998.

Anon. 2003. “Learning curve”, Building Services Journal, September 2003.

ASHRAE. 2001a. Ventilation for Acceptable Indoor Air Quality. ASHRAE Standard 62; American Society of Heating, Refrigerating and Air-Conditioning Engineers, Inc.

ASHRAE. 2001b. Energy Standard for Buildings Except Low-Rise Residential Buildings. ASHRAE Standard 90.1; American Society of Heating, Refrigerating and Air-Conditioning Engineers, Inc.

ASHRAE. 2001c. Handbook of Fundamentals. American Society of Heating, Refrigerating and AirConditioning Engineers, Inc.

ASHRAE. 2001d. Energy-Efficient Design of Low-Rise Residential Buildings. ASHRAE Standard 90.2; American Society of Heating, Refrigerating and Air-Conditioning Engineers, Inc.

Ask, A. 2003. "Ventilation and Air Leakage”, ASHRAE Journal Vol. 45, No. 11.

ASTM. 1999. E779-99, Standard Test Method for Determining Air Leakage Rate by Fan Pressurization. American Society for Testing and Materials.

Bombaru, Jutras, and Patenaude. 1988. “The Air Permeance of Building Materials”, Canada Mortgage and Housing Corporation.

Briggs, R., Crawley, D., and J.S. Schliesing. 1992. Energy requirements for office buildings. Volume 1, Existing buildings. GRI-90/0236.1 by Battelle, Pacific Northwest Laboratory, for Gas Research Institute.

CGSB. 1986. CAN/CGSB-149.10-86, Determination of the airtightness of building envelopes by the fan depressurization method using the building's air handling systems. Canadian General Standards Board.

CGSB. 1996. CAN/CGSB-149.15-96, Determination of the overall envelope airtightness of buildings by the fan depressurization method using the building's air handling systems. Canadian General Standards Board.

CIBSE. 2000. Technical Memoranda TM23:2000, Testing Buildings for Air Leakage. Chartered Institution of Building Services Engineers.

Cohen, R. 2003. “Tightly knit.” Building Services Journal, June 2003.

Crawley, D., and J. Schliesing. 1992. Energy requirements for office buildings. Volume 2, Recent and future buildings. GRI-90/0236.2 by Battelle, Pacific Northwest Laboratory, for Gas Research Institute.

Diamond, R.C., Modera, M.P., and H.E. Feustel. 1986. "Ventilation and Occupant Behavior in Two Apartment Buildings," in, Proceedings, The 7th Air Infiltration Centre Conference on Occupant Interaction with Ventilation Systems, Stratford, UK.

Dols, W.S. and G.W. Walton. 2002. CONTAMW 2.0 User Manual, NISTIR 6921. 
Edwards, C. 1999. Modelling of Ventilation and Infiltration Energy Impacts in Mid- and High-Rise Apartment Buildings. Report by Sheltair Scientific Ltd. For Canada Mortgage and Housing Corporation.

Emmerich, S., Persily, A. and D. VanBronkhorst. 1995. A workplan to analyze the energy impacts of envelope airtightness in office buildings. NSITIR 5758, National Institute of Standards and Technology.

Emmerich, S., and A. Persily. 1998. "Energy Impacts of Infiltration and Ventilation in U.S. Office Buildings Using Multizone Airflow Simulation.” IAQ and Energy '98, American Society of Heating, Refrigerating, and Air-Conditioning Engineers, Inc.

Feustel, H.E. and R.C. Diamond. 1996. "Diagnostics and Measurements of Infiltration and Ventilation Systems in High-Rise Apartment Buildings", in Proceedings of the ACEEE Summer Study on Energy Efficiency in Buildings.

HFG. 2004. Air Barrier Study. Hanscomb Faithful and Gould International, prepared for Shepley Bulfinch Richardson and Abbott.

ISO. 1996. Standard 9972, Thermal Insulation - Determination of Building Airtightness - Fan

Pressurization Method. International Standards Organization.

Kennett. 2004. “Relocation, relocation.” Building Services Journal, June 2004.

Klein S. TRNSYS - A Transient System Simulation Program Engineering Experiment Station Report 38-13. (2000) Solar Energy Laboratory, University of Wisconsin - Madison.

Marion,W. and K. Urban. 1995. User’s Manual for TMY2s. National Renewable Energy Laboratory.

McBride, M.F. 1995. "Development of Economic Scalar Ratios for ASHRAE Standard 90.1R”, Proceedings of Thermal Performance of the Exterior Envelopes of Buildings VI, ASHRAE.

McDowell, T., Emmerich, S., Thornton, J., and G. Walton. 2003. "Integration of Airflow and Energy Simulation Using CONTAM and TRNSYS.” ASHRAE Transactions 2003.

Modera, M.P., Brunsell, J.T. and R. C. Diamond. 1985. "Improving Diagnostics and Energy

Analysis for Multifamily Buildings: A Case Study," in Proceedings, of the Thermal Performance of the Exterior Envelopes of Buildings, Clearwater, Florida.

Olivier, D. 2001. “Shattering the energy barrier” Building Services Journal: April 2001.

Parekh, A. 1992 "Power Demand and Energy Savings Through Air Leakage Control in High-Rise Residential Buildings in Cold Climates”. 1992. Proceedings of Thermal Performance of the Exterior Envelope of Buildings V.

Persily, A.K. 1993. Envelope Design Guidelines for Office Buildings: Thermal Integrity and Airtightness. NISTIR 4821, National Institute of Standards and Technology.

Persily, A.K. 1998. “Airtightness of Commercial and Institutional Buildings” Proceedings of ASHRAE Thermal Envelopes VII Conference.

Potter, I., Jones, T., and W. Booth. 1995. TechNote 8/95 Air Leakage of Office Buildings. Building Services Research and Information Association.

Proskiw, G. and B. Phillips. 2001. Air Leakage Characteristics, Test Methods and Specifications for Large Buildings. Canada Mortgage and Housing Corporation research report. 
Scanada. 1991. Development of Design Procedures and Guidelines for Reducing Electrical Demand by Air Leakage Control in High-Rise Residential Buildings, CMHC Final Report.

Shaw, C. Y., and J. T. Reardon. 1995. “Changes in Airtightness Levels of Six Office Buildings” ASTM STP 1255, American Society of Testing and Materials.

Spinu, M. 2004. E-mail to Wagdy Anis dated 06/18/2004.

Synertech Systems Corporation. 1987. "Integrating Analytical Tactics into New York State's Weatherization Assistance Program: Project Findings," Albany, New York: New York State Energy and Research Development Authority.

TWC. 2004. "Prototype Air Barrier Buildings” The Waterproofing Company, Boston, MA.

Walton G. 1989. AIRNET - A Computer Program for Building Airflow Network Modeling. NISTIR 894072. National Institute of Standards and Technology.

Wray, C., Theaker, I., and P. Moffatt. 1998. "Field Testing to Characterise Suite Ventilation in Recently Constructed Mid- and High-Rise Residential Buildings", CMHC Project Report. 


\section{Appendix A Commercial Building Airtightness Data}

Table A1 summarizes the measured whole building envelope airtightness data that was considered in developing the airtightness levels for this study. The majority of this data was previously reviewed and analyzed by Persily (1998), however some additional buildings were added. The air leakage values in the table are normalized by the area of the above-ground portion of the building envelope. Information on year of construction, building construction type, number of stories, or building floor area was not available for all buildings.

Table A1 Summary Table of Measured Nonresidential Building Envelope Airtightness Data

\begin{tabular}{|c|c|c|c|c|c|c|c|c|}
\hline Building & Building type & $\begin{array}{c}\text { Year of } \\
\text { construction }\end{array}$ & \begin{tabular}{|c|} 
Construction \\
type
\end{tabular} & Stories & Floor area & $\begin{array}{r}\text { Air le } \\
\text { at } 7 \\
(0.3 \mathrm{i}\end{array}$ & $\begin{array}{l}\text { kage } \\
\text { Pa } \\
\text { w.g.) }\end{array}$ & Ref \\
\hline & & & & & $\mathrm{m}^{2}$ & $\mathrm{~L} / \mathrm{s}-\mathrm{m}^{2}$ & $\mathrm{cfm} / \mathrm{ft}^{2}$ & \\
\hline Anchorage & Office & 1981 & Concrete panel & 6 & 48500 & 3.8 & 0.75 & 1 \\
\hline Ann Arbor & Office & 1975 & Masonry & 4 & 5300 & 2.3 & 0.46 & 1 \\
\hline Columbia & Office & 1977 & Concrete panel & 15 & 21600 & 3.4 & 0.67 & 1 \\
\hline Huron & Office & 1978 & Masonry & 4 & 6900 & 1.1 & 0.21 & 1 \\
\hline Norfolk & Office & 1978 & Masonry & 8 & 18600 & 4.1 & 0.80 & 1 \\
\hline Pittsfield & Office & 1977 & Masonry & 2 & 1800 & 2.0 & 0.39 & 1 \\
\hline Springfield & Office & 1982 & Concrete panel & 5 & 14600 & 5.2 & 1.03 & 1 \\
\hline Overland & Office & 1990 & Curtain & 5 & 35100 & 12.0 & 2.37 & 7 \\
\hline Albany & School & $<1992$ & & 3 & 22300 & 3.1 & 0.60 & 2 \\
\hline Admin & School & $<1992$ & & 3 & 8200 & 0.7 & 0.15 & 2 \\
\hline Argentine & School & $<1992$ & & 3 & 700 & 2.9 & 0.57 & 2 \\
\hline Bishop Ryan & School & $<1992$ & & 3 & 5600 & 1.8 & 0.36 & 2 \\
\hline CLC & School & $<1992$ & & 3 & 4600 & 0.9 & 0.17 & 2 \\
\hline Green Mtn. & School & $<1992$ & & 3 & 2400 & 3.3 & 0.66 & 2 \\
\hline Green Gym & School & $<1992$ & & 3 & 900 & 4.1 & 0.80 & 2 \\
\hline Laurel & School & $<1992$ & & 3 & 1500 & 1.0 & 0.19 & 2 \\
\hline Middle & School & $<1992$ & & 3 & 7200 & 4.1 & 0.80 & 2 \\
\hline S. Pines & School & $<1992$ & & 3 & 4400 & 1.0 & 0.19 & 2 \\
\hline S. Tama & School & $<1992$ & & 3 & 700 & 2.3 & 0.45 & 2 \\
\hline Russell & School & $<1992$ & & 3 & 3300 & 3.0 & 0.59 & 2 \\
\hline Velva & School & $<1992$ & & 3 & 5600 & 2.7 & 0.54 & 2 \\
\hline NRCC-A & Office & 1970 & Concrete panel & 9 & & 6.2 & 1.23 & 3 \\
\hline NRCC-B & Office & 1964 & Concrete panel & 17 & & 3.1 & 0.61 & 3 \\
\hline NRCC-C & Office & 1970 & Concrete panel & 20 & & 2.6 & 0.61 & 3 \\
\hline NRCC-D & Office & 1971 & Curtain & 20 & & 3.4 & 0.67 & 3 \\
\hline NRCC-E & Office & 1968 & Curtain & 21 & & 2.0 & 0.39 & 3 \\
\hline NRCC-F & Office & 1973 & Concrete panel & 16 & & 2.0 & 0.39 & 3 \\
\hline NRCC-G & Office & 1974 & Concrete panel & 25 & & 2.8 & 0.55 & 3 \\
\hline NRCC-H & Office & 1974 & Curtain & 20 & & 1.4 & 0.27 & 3 \\
\hline NRCC-S-A & School & 1970 & Concrete panel & 1 & 2700 & 9.0 & 1.77 & 4 \\
\hline NRCC-S-B & School & 1971 & Masonry & 1 & 1900 & 8.2 & 1.61 & 4 \\
\hline NRCC-S-C & School & 1965 & Masonry & 1 & 3800 & 7.4 & 1.45 & 4 \\
\hline
\end{tabular}




\begin{tabular}{|c|c|c|c|c|c|c|c|}
\hline NRCC-S-D & School & 1973 & Masonry & 1 & 3500 & 12.3 & 2.41 \\
\hline NRCC-S-E & School & 1957 & Masonry & 1 & 3700 & 7.1 & 1.41 \\
\hline NRCC-S-F & School & 1952 & Masonry & 1 & 3100 & 6.3 & 1.25 \\
\hline NRCC-S-G & School & 1968 & Masonry & 1 & 5400 & 5.9 & 1.17 \\
\hline NRCC-S-H & School & 1965 & Masonry & 1 & 5200 & 4.9 & 0.96 \\
\hline NRCC-S-I & School & 1968 & Masonry & 1 & 2600 & 11.4 & 2.25 \\
\hline NRCC-S-J & School & 1972 & Masonry & 1 & 3000 & 8.4 & 1.65 \\
\hline NRCC-S-K & School & 1968 & Masonry & 1 & 3200 & 5.5 & 1.09 \\
\hline NRCC-BH & Retail & 1957 & Masonry & 3 & & 9.0 & 1.77 \\
\hline NRCC-CK & Retail & 1963 & Masonry & 3 & & 6.9 & 1.37 \\
\hline NRCC-HC & Retail & 1978 & Masonry & 3 & & 19.8 & 3.90 \\
\hline NRCC-MD & Retail & 1977 & Masonry & 3 & & 18.4 & 3.62 \\
\hline NRCC-MK & Retail & 1967 & Masonry & 3 & & 9.4 & 1.85 \\
\hline NRCC-MS & Retail & 1955 & Masonry & 3 & & 17.0 & 3.34 \\
\hline NRCC-OD & Retail & 1979 & Concrete panel & 3 & & 17.4 & 3.42 \\
\hline NRCC-PO & Retail & 1979 & Concrete panel & 3 & & 14.1 & 2.77 \\
\hline NRCC-RM & Retail & 1957 & Masonry & 3 & & 5.7 & 1.13 \\
\hline NRCC-WG & Retail & 1954 & Masonry & 3 & & 19.4 & 3.82 \\
\hline Parekh1 & Apartment & $<1992$ & & 21 & & 2.4 & 0.47 \\
\hline Parekh2 & Apartment & $<1992$ & & 10 & & 2.1 & 0.41 \\
\hline NRCC-R & Apartment & 1981 & Masonry & 5 & & 4.9 & 0.96 \\
\hline BRE-1 & Office & 1980 & Masonry & & & 3.1 & 0.61 \\
\hline BRE-2 & Office & 1963 & Masonry & & & 3.0 & 0.59 \\
\hline BRE-3 & Office & 1991 & Masonry & & & 3.1 & 0.61 \\
\hline BRE-4 & Office & 1965 & Masonry & & & 6.7 & 1.32 \\
\hline BRE-5 & Office & 1987 & Masonry & & & 3.8 & 0.75 \\
\hline BRE-6 & Office & 1990 & Masonry & & & 5.1 & 1.01 \\
\hline BRE-7 & Office & 1990 & Masonry & & & 8.7 & 1.71 \\
\hline BRE-8 & Office & 1971 & Concrete panel & & & 9.5 & 1.88 \\
\hline BRE-9 & Office & 1986 & Masonry & & & 10.2 & 2.00 \\
\hline BRE-10 & Office & 1985 & Concrete panel & & & 11.6 & 2.28 \\
\hline BSRIA-1 & OfficeNV & 1970 & Prefab panels & 2 & & 3.7 & 0.72 \\
\hline BSRIA-2 & OfficeNV & Elizabethan & Masonry & 3 & & 4.3 & 0.83 \\
\hline BSRIA-3 & Office & 1991 & $\begin{array}{l}\text { Steelframe w/ } \\
\text { masonry }\end{array}$ & 6 & & 4.4 & 0.85 \\
\hline BSRIA-4 & Office & 1987.5 & $\begin{array}{l}\text { Steelframe w/ } \\
\text { masonry }\end{array}$ & 2 & & 10.9 & 2.14 \\
\hline BSRIA-5 & OfficeNV & 1963 & $\begin{array}{l}\text { Concrete } \\
\text { panels on } \\
\text { frame }\end{array}$ & 7 & & 5.4 & 1.07 \\
\hline BSRIA-6 & Office & 1991 & $\begin{array}{l}\text { Steelframe w/ } \\
\text { masonry }\end{array}$ & 3 & & 8.5 & 1.67 \\
\hline BSRIA-7 & Office & 1986 & $\begin{array}{l}\text { Steelframe w/ } \\
\text { masonry }\end{array}$ & 6 & & 14.5 & 2.84 \\
\hline BSRIA-8 & Office & 1989 & $\begin{array}{c}\text { Curtain } \\
\text { wall/brick }\end{array}$ & 5 & & 4.3 & 0.85 \\
\hline
\end{tabular}




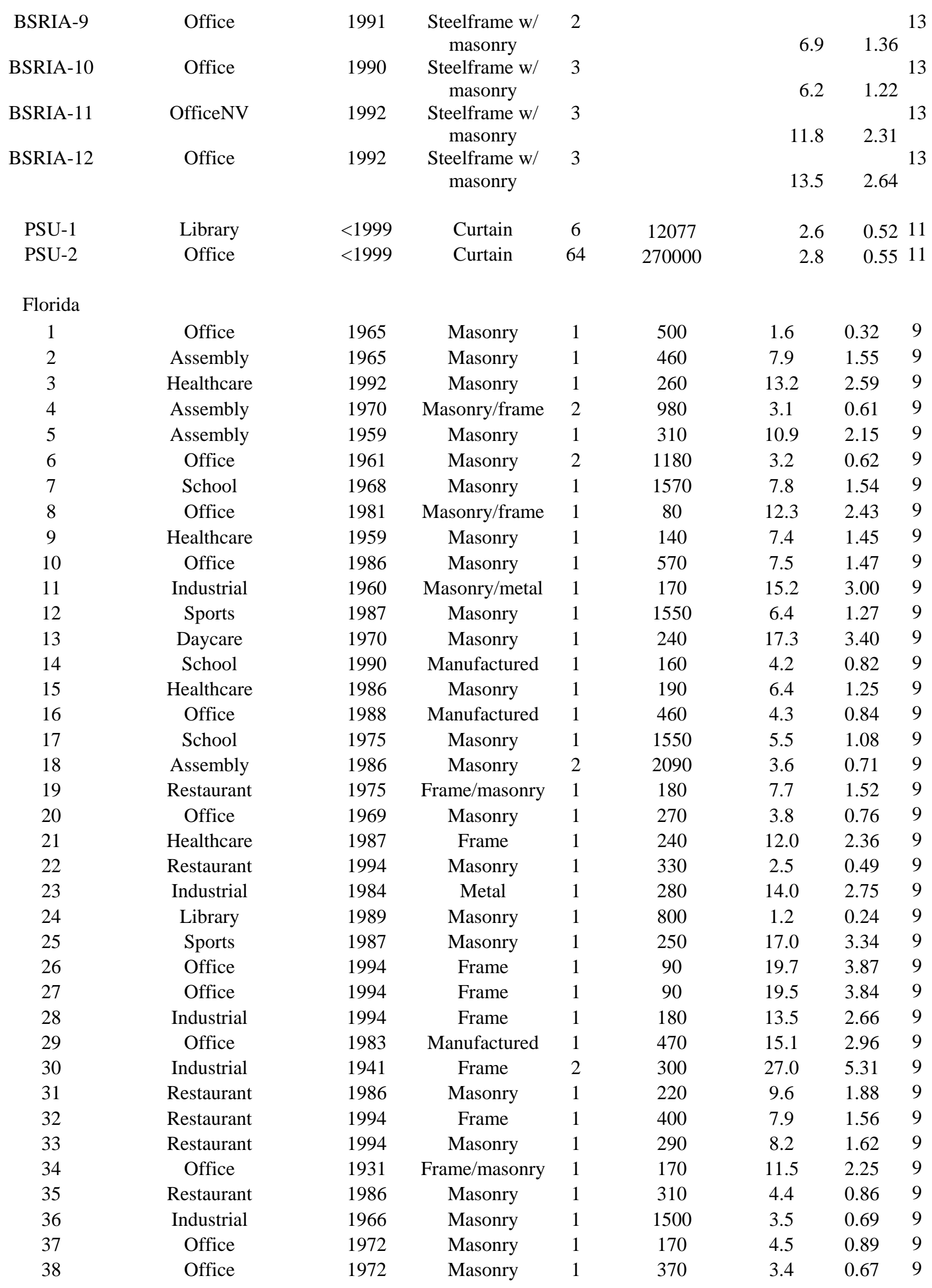




\begin{tabular}{|c|c|c|c|c|c|c|c|}
\hline 39 & Office & 1946 & Masonry & 1 & 200 & 9.6 & 1.89 \\
\hline 40 & School & 1966 & Frame/masonry & 1 & 230 & 15.0 & 2.95 \\
\hline 41 & Animal & 1966 & Frame/masonry & 1 & 70 & 11.8 & 2.32 \\
\hline 42 & Office & 1966 & Frame/masonry & 1 & 590 & 14.8 & 2.91 \\
\hline 43 & Bar & 1966 & Frame/masonry & 1 & 200 & 20.7 & 4.08 \\
\hline 44 & Office & 1966 & Frame/masonry & 1 & 120 & 17.5 & 3.44 \\
\hline 45 & Industrial & 1966 & Frame/masonry & 1 & 240 & 12.5 & 2.46 \\
\hline 46 & Office & 1966 & Frame/masonry & 1 & 350 & 34.6 & 6.81 \\
\hline 47 & Sports & 1966 & Frame/masonry & 1 & 90 & 12.7 & 2.51 \\
\hline 49 & Retail & 1966 & Frame/masonry & 1 & 90 & 8.9 & 1.75 \\
\hline 50 & Retail & 1966 & Frame/masonry & 1 & 90 & 20.8 & 4.10 \\
\hline 51 & Office & 1951 & Masonry & 1 & 500 & 6.6 & 1.30 \\
\hline 52 & Office & 1964 & Masonry & 1 & 170 & 3.4 & 0.66 \\
\hline 53 & Industrial & 1986 & Metal & 1 & 260 & 3.5 & 0.69 \\
\hline 54 & Office & 1976 & Masonry & 1 & 240 & 11.6 & 2.29 \\
\hline 55 & Industrial & 1978 & Metal & 1 & 930 & 8.6 & 1.69 \\
\hline 56 & Industrial & 1983 & Metal & 1 & 1150 & 5.6 & 1.10 \\
\hline 57 & Sports & 1982 & Masonry & 1 & 660 & 15.3 & 3.00 \\
\hline 58 & Retail & 1994 & Masonry & 1 & 430 & 3.3 & 0.64 \\
\hline 59 & Retail & 1973 & Masonry & 1 & 150 & 9.9 & 1.95 \\
\hline 60 & Office & 1985 & Manufactured & 1 & 80 & 4.3 & 0.86 \\
\hline 61 & Office & 1983 & Manufactured & 1 & 120 & 9.0 & 1.78 \\
\hline 62 & Restaurant & 1963 & Frame & 1 & 730 & 5.3 & 1.04 \\
\hline 63 & Office & 1990 & Masonry & 1 & 620 & 2.1 & 0.40 \\
\hline 64 & School & 1965 & Masonry & 1 & 940 & 3.1 & 0.61 \\
\hline 65 & School & 1965 & Masonry & 1 & 190 & 3.0 & 0.59 \\
\hline 66 & School & 1965 & Masonry & 1 & 470 & 9.1 & 1.79 \\
\hline 67 & Hotel & 1977 & Masonry & 1 & 1400 & 6.7 & 1.32 \\
\hline 68 & Hotel & 1977 & Masonry & 2 & 1180 & 6.3 & 1.24 \\
\hline 69 & Retail & 1989 & Masonry & 1 & 400 & 10.9 & 2.16 \\
\hline 70 & Retail & 1969 & Masonry & 1 & 230 & 1.1 & 0.22 \\
\hline \multicolumn{8}{|c|}{ FSEC2 } \\
\hline 1 & ELEM. SCHOOL 1 & 1966 & MASONRY & 1 & 4657.9 & 40.0 & 7.85 \\
\hline 2 & CHICKEN REST. & 1993 & MASONRY & 1 & 293.66 & 3.7 & 0.73 \\
\hline 3 & COMMUNITY CENTER & 1991 & MET/FRAME & 1 & 2223.5 & 101.6 & 19.93 \\
\hline 4 & ELEM. SCHOOL 2 & 1998 & MET/MAS & 1 & 464.5 & 41.8 & 8.21 \\
\hline 5 & ELEM. SCHOOL 3 & 1998 & MET/MAS & 1 & 464.5 & 46.5 & 9.13 \\
\hline 6 & SEAFOOD REST. & 1998 & FRAME & 1 & 762.15 & 28.2 & 5.54 \\
\hline 7 & RIVER FRONT REST. & 1986 & MASONRY & 2 & 543.47 & 3.8 & 0.74 \\
\hline 8 & STEAKHOUSE REST. & 1995 & MET/FRAME & 1 & 568.08 & 29.1 & 5.70 \\
\hline 9 & MDL. SCHOOL 1 B & 1996 & FRAME & 1 & 65.587 & 6.1 & 1.20 \\
\hline 10 & MDL. SCHOOL 1 SW & 1991 & FRAME & 1 & 60.385 & 3.9 & 0.77 \\
\hline 11 & MDL. SCHOOL 1 D & 1991 & FRAME & 1 & 70.232 & 5.3 & 1.05 \\
\hline 12 & MDL. SCHOOL 1 E & 1991 & FRAME & 1 & 70.232 & 11.6 & 2.27 \\
\hline 13 & MDL. SCHOOL 21 & 1997 & FRAME & 1 & 66.888 & 12.0 & 2.36 \\
\hline 14 & MDL. SCHOOL 26 & 1997 & FRAME & 1 & 66.888 & 13.9 & 2.72 \\
\hline 15 & MDL. SCHOOL 27 & 1997 & FRAME & 1 & 66.888 & 19.9 & 3.89 \\
\hline 16 & BAR AND GRILL & 1985 & MASONRY & 1 & 222.96 & 9.5 & 1.87 \\
\hline 17 & CONVEN. STORE & 1988 & MASONRY & 1 & 401.33 & 10.1 & 1.98 \\
\hline 18 & OCEAN FRONT REST. & 1962 & FRAME & 1 & 555.26 & 27.3 & 5.36 \\
\hline
\end{tabular}


19

20

Overall mean

Note: Not all parameters were reported for some buildings.
1960 MASONRY $\quad 1 \quad 139.35$

1984 MET/MAS $1 \quad 347.54$
35.7

19.9

9.7
6.9912

3.9112

1.9

\section{References for Measured Airtightness Data}

1. Persily, A.K. and R.A. Grot. 1986. "Pressurization Testing of Federal Buildings," Measured Air Leakage of Buildings, ASTM STP 904, H.R. Treschsel and P.L. Lagus, Eds., American Society for Testing and Materials, Philadelphia, pp. 184-200.

2. Brennan, T., Turner, W., Fisher, G., Thompson, B., and B. Ligman. 1992. "Fan Pressurization of School Buildings," Thermal Performance of the Exterior Envelopes of Buildings V, ASHRAE, Atlanta, pp. 643-645.

3. Tamura, G.T. and C.Y. Shaw. 1976. "Studies on Exterior Wall Air Tightness and Air Infiltration of Tall Buildings," ASHRAE Transactions, Vol. 82 (II), pp. 122-34.

4. Shaw, C.Y. and L. Jones. 1979. "Air Tightness and Air Infiltration of School Buildings," ASHRAE Transactions, Vol.85, Part 1, pp. 85-95.

5. Shaw. C.Y. 1981. "Air Tightness: Supermarkets and Shopping Malls," ASHRAE Journal, March 1981, pp.44-46

6. Shaw, C.Y., Magee R.J. and J. Rousseau. 1991. "Overall and Component Airtightness Values of a FiveStory Apartment Building," ASHRAE Transactions, Vol.97, Part 2, pp.347-353.

7. Persily, A.K., Dols, W.S., Nabinger, S.J. and S. Kirchner. 1991. "Preliminary Results of the Environmental Evaluation of the Federal Records Center in Overland Missouri," NISTIR 4634.

8. Lundin, L.I. 1986. "Air Leakage in Industrial Buildings - Description of Equipment," Measured Air Leakage of Buildings, ASTM STP 904, H.R. Treschsel and P.L. Lagus, Eds., American Society for Testing and Materials, Philadelphia, pp. 101-105.

9. Cummings, J.B., Withers, C.R., Moyer, N., Fairey, P., and B. McKendry. 1996. Uncontrolled Air Flow in Non-Residential Buildings, FSEC-CR-878-96, Florida Solar Energy Center.

10. Perera, M.D.A.E.S., Henderson, J., and B.C. Webb. 1997. Simple Air Leakage Predictor for Office Buildings: Assessing Envelope Airtightness During Design or Before Refurbishment. CIBSE National Conference.

11. Bahnfleth, W.P., Yuill, G.K. and B.W. Lee. 1999. "Protocol for Field Testing of Tall Buildings to Determine Envelope Air Leakage Rate," ASHRAE Transactions, Vol.105 (2).

12. Cummings, J.B., Shirey, D.B., Withers, C., Raustad, R. and N. Moyer. 2000. Evaluating the Impacts of Uncontrolled Air Flow and HVAC Performance Problems on Florida's Commercial and Institutional Buildings, Final Report, FSEC-CR-1210-00.

13. Potter, I., Jones, T., and W. Booth. 1995. TechNote 8/95 Air Leakage of Office Buildings. Building Services Research and Information Association.

14. Parekh, A. 1992. 'Power Demand and Energy Savings Through Air Leakage Control in High-Rise Residential Buildings in Cold Climates'. Proceedings of Thermal Performance of the Exterior Envelope of Buildings V. 